\title{
Nonsingularity Conditions for FB System of Reformulating Nonlinear Second-Order Cone Programming
}

\author{
Shaohua Pan, ${ }^{1}$ Shujun $\mathrm{Bi}^{1}{ }^{1}$ and Jein-Shan Chen ${ }^{2}$ \\ ${ }^{1}$ Department of Mathematics, South China University of Technology, Guangzhou 510641, China \\ ${ }^{2}$ Mathematics Division, National Center for Theoretical Sciences, Taipei 11677, Taiwan
}

Correspondence should be addressed to Jein-Shan Chen; jschen@math.ntnu.edu.tw

Received 2 June 2012; Accepted 9 December 2012

Academic Editor: Narcisa C. Apreutesei

Copyright (c) 2013 Shaohua Pan et al. This is an open access article distributed under the Creative Commons Attribution License, which permits unrestricted use, distribution, and reproduction in any medium, provided the original work is properly cited.

\begin{abstract}
This paper is a counterpart of Bi et al., 2011. For a locally optimal solution to the nonlinear second-order cone programming (SOCP), specifically, under Robinson's constraint qualification, we establish the equivalence among the following three conditions: the nonsingularity of Clarke's Jacobian of Fischer-Burmeister (FB) nonsmooth system for the Karush-Kuhn-Tucker conditions, the strong second-order sufficient condition and constraint nondegeneracy, and the strong regularity of the Karush-Kuhn-Tucker point.
\end{abstract}

\section{Introduction}

The nonlinear second-order cone programming (SOCP) problem can be stated as

$$
\begin{array}{ll}
\min _{\zeta \in \mathbb{R}^{n}} & f(\zeta) \\
\text { s.t. } & h(\zeta)=0, \\
& g(\zeta) \in \mathscr{K},
\end{array}
$$

where $f: \mathbb{R}^{n} \rightarrow \mathbb{R}, h: \mathbb{R}^{n} \rightarrow \mathbb{R}^{m}$, and $g: \mathbb{R}^{n} \rightarrow \mathbb{R}^{n}$ are given twice continuously differentiable functions, and $\mathscr{K}$ is the Cartesian product of some second-order cones, that is,

$$
\mathscr{K}:=\mathscr{K}^{n_{1}} \times \mathscr{K}^{n_{2}} \times \cdots \times \mathscr{K}^{n_{r}},
$$

with $n_{1}+\cdots+n_{r}=n$ and $\mathscr{K}^{n_{j}}$ being the second-order cone (SOC) in $\mathbb{R}^{n_{j}}$ defined by

$$
\mathscr{K}^{n_{j}}:=\left\{\left(x_{j 1}, x_{j 2}\right) \in \mathbb{R} \times \mathbb{R}^{n_{j}-1} \mid x_{j 1} \geq\left\|x_{j 2}\right\|\right\} .
$$

By introducing a slack variable to the second constraint, the SOCP (1) is equivalent to

$$
\begin{array}{rl}
\min _{\zeta, x \in \mathbb{R}^{n}} & f(\zeta) \\
\text { s.t. } & h(\zeta)=0, \\
& g(\zeta)-x=0, \quad x \in \mathscr{K} .
\end{array}
$$

In this paper, we will concentrate on this equivalent formulation of problem (1).

Let $L: \mathbb{R}^{n} \times \mathbb{R}^{n} \times \mathbb{R}^{m} \times \mathbb{R}^{n} \times \mathscr{K} \rightarrow \mathbb{R}$ be the Lagrangian function of problem (4)

$$
L(\zeta, x, \mu, s, y):=f(\zeta)+\langle\mu, h(\zeta)\rangle+\langle g(\zeta)-x, s\rangle-\langle x, y\rangle,
$$

and denote by $\mathcal{N}_{\mathscr{K}}(x)$ the normal cone of $\mathscr{K}$ at $x$ in the sense of convex analysis [1]:

$$
\mathcal{N}_{\mathscr{K}}(x)= \begin{cases}\left\{d \in \mathbb{R}^{n}:\langle d, z-x\rangle \leq 0 \forall z \in \mathscr{K}\right\}, & \text { if } x \in \mathscr{K}, \\ \emptyset, & \text { if } x \notin \mathscr{K} .\end{cases}
$$


Then the Karush-Kuhn-Tucker (KKT) conditions for (4) take the following form:

$$
\begin{gathered}
\mathscr{J}_{\zeta, x} L(\zeta, x, \mu, s, y)=0, \quad h(\zeta)=0, \\
g(\zeta)-x=0, \quad-y \in \mathcal{N}_{\mathscr{K}}(x),
\end{gathered}
$$

where $\mathscr{J}_{\zeta, x} L(\zeta, x, \mu, s, y)$ is the derivative of $L$ at $(\zeta, x, \mu, s, y)$ with respect to $(\zeta, x)$. Recall that $\phi^{\text {soc }}$ is an SOC complementarity function associated with the cone $\mathscr{K}$ if

$$
\begin{gathered}
\phi^{\text {soc }}(x, y)=0 \Longleftrightarrow x \in \mathscr{K}, y \in \mathscr{K}, \\
\langle x, y\rangle=0 \Longleftrightarrow-y \in \mathscr{N}_{\mathscr{K}}(x) .
\end{gathered}
$$

With an SOC complementarity function $\phi^{\text {soc }}$ associated with $\mathscr{K}$, we may reformulate the KKT optimality conditions in (7) as the following nonsmooth system:

$$
E(\zeta, x, \mu, s, y):=\left[\begin{array}{c}
\mathscr{J}_{\zeta, x} L(\zeta, x, \mu, s, y) \\
h(\zeta) \\
g(\zeta)-x \\
\phi^{\text {soc }}(x, y)
\end{array}\right]=0 .
$$

The most popular SOC complementarity functions include the vector-valued natural residual (NR) function and Fischer-Burmeister (FB) function, respectively, defined as

$$
\begin{gathered}
\phi_{\mathrm{NR}}(x, y):=x-\Pi_{\mathscr{K}}(x-y), \quad \forall x, y \in \mathbb{R}^{n}, \\
\phi_{\mathrm{FB}}(x, y):=(x+y)-\sqrt{x^{2}+y^{2}}, \quad \forall x, y \in \mathbb{R}^{n},
\end{gathered}
$$

where $\Pi_{\mathscr{K}}(\cdot)$ is the projection operator onto the closed convex cone $\mathscr{K}, x^{2}=x \circ x$ means the Jordan product of $x$ and itself, and $\sqrt{x}$ denotes the unique square root of $x \in \mathscr{K}$. It turns out that the FB SOC complementarity function $\phi_{\mathrm{FB}}$ enjoys almost all favorable properties of the NR SOC complementarity function $\phi_{\mathrm{NR}}$ (see [2]). Also, the squared norm of $\phi_{\mathrm{FB}}$ induces a continuously differentiable merit function with globally Lipschitz continuous derivative $[3,4]$. This greatly facilitates the globalization of the semismooth Newton method $[5,6]$ for solving the FB nonsmooth system of KKT conditions:

$$
E_{\mathrm{FB}}(\zeta, x, \mu, s, y):=\left[\begin{array}{c}
\mathscr{J}_{\zeta, x} L(\zeta, x, \mu, s, y) \\
h(\zeta) \\
g(\zeta)-x \\
\phi_{\mathrm{FB}}(x, y)
\end{array}\right]=0 .
$$

Recently, with the help of [7, Theorem 30] and [8, Lemma 11], Wang and Zhang [9] gave a characterization for the strong regularity of the KKT point of the SOCP (1) via the nonsingularity study of Clarke's Jacobian of the NR nonsmooth system

$$
E_{\mathrm{NR}}(\zeta, x, \mu, s, y):=\left[\begin{array}{c}
\mathscr{J}_{\zeta, x} L(\zeta, x, \mu, s, y) \\
h(\zeta) \\
g(\zeta)-x \\
\phi_{\mathrm{NR}}(x, y)
\end{array}\right]=0 .
$$

They showed that the strong regularity of the KKT point, the nonsingularity of Clarke's Jacobian of $E_{\mathrm{NR}}$ at the KKT point, and the strong second-order sufficient condition and constraint nondegeneracy [7] are all equivalent. These nonsingularity conditions are better structured than those of [10] for the nonsingularity of the $B$-subdifferential of the NR system. Then, it is natural to ask the following: is it possible to obtain a characterization for the strong regularity of the KKT point by studying the nonsingularity of Clarke's Jacobian of $E_{\mathrm{FB}}$. Note that up till now one even does not know whether the $B$-subdifferential of the FB system is nonsingular or not without the strict complementarity assumption.

In this work, for a locally optimal solution to the nonlinear SOCP (4), under Robinson's constraint qualification, we show that the strong second-order sufficient condition and constraint nondegeneracy introduced in [7], the nonsingularity of Clarke's Jacobian of $E_{\mathrm{FB}}$ at the KKT point, and the strong regularity of the KKT point are equivalent to each other. This, on the one hand, gives a new characterization for the strong regularity of the KKT point and, on the other hand, provides a mild condition to guarantee the quadratic convergence rate of the semismooth Newton method $[5,6]$ for the FB system. Note that parallel results are obtained recently for the FB system of the nonlinear semidefinite programming (see [11]); however, we do not duplicate them. As will be seen in Sections 3 and 4 , the analysis techniques here are totally different from those in [11]. It seems hard to put them together in a unified framework under the Euclidean Jordan algebra. The main reason causing this is due to completely different analysis when dealing with the Clarke Jacobians associated with FB SOC complementarity function and FB semidefinite cone complementarity function.

Throughout this paper, $I$ denotes an identity matrix of appropriate dimension, $\mathbb{R}^{n}(n>1)$ denotes the space of $n$ dimensional real column vectors, and $\mathbb{R}^{n_{1}} \times \cdots \times \mathbb{R}^{n_{r}}$ is identified with $\mathbb{R}^{n_{1}+\cdots+n_{r}}$. Thus, $\left(x_{1}, \ldots, x_{r}\right) \in \mathbb{R}^{n_{1}} \times \cdots \times \mathbb{R}^{n_{r}}$ is viewed as a column vector in $\mathbb{R}^{n_{1}+\cdots+n_{r}}$. The notations int $\mathscr{K}^{n}$, bd $\mathscr{K}^{n}$, and $\mathbf{b d}^{+} \mathscr{K}^{n}$ denote the interior, the boundary, and the boundary excluding the origin of $\mathscr{K}^{n}$, respectively. For any $x \in \mathbb{R}^{n}$, we write $x \succeq_{\mathscr{K}^{n}} 0$ (resp., $x>_{\mathscr{K}^{n}} 0$ ) if $x \in \mathscr{K}^{n}$ (resp., $x \in$ int $\mathscr{K}^{n}$ ). For any given real symmetric matrix $A$, we write $A \geq 0$ (resp., $A>0$ ) if $A$ is positive semidefinite (resp., positive definite). In addition, $\mathscr{J}_{\omega} f(\omega)$ and $\mathscr{F}_{\omega \omega}^{2} f(\omega)$ denote the derivative and the second-order derivative, respectively, of a twice differentiable function $f$ with respect to the variable $\omega$.

\section{Preliminary Results}

First we recall from [12] the definition of Jordan product and spectral factorization.

Definition 1. The Jordan product of $x=\left(x_{1}, x_{2}\right), y=\left(y_{1}\right.$, $\left.y_{2}\right) \in \mathbb{R} \times \mathbb{R}^{n-1}$ is given by

$$
x \circ y:=\left(\langle x, y\rangle, x_{1} y_{2}+y_{1} x_{2}\right) \text {. }
$$

Unlike scalar or matrix multiplication, the Jordan product is not associative in general. The identity element under this product is $e:=(1,0, \ldots, 0)^{T} \in \mathbb{R}^{n}$, that is, $e \circ x=x$ for all 
$x \in \mathbb{R}^{n}$. For each $x=\left(x_{1}, x_{2}\right) \in \mathbb{R} \times \mathbb{R}^{n-1}$, we define the associated arrow matrix by

$$
L_{x}:=\left[\begin{array}{ll}
x_{1} & x_{2}^{T} \\
x_{2} & x_{1} I
\end{array}\right] \text {. }
$$

Then it is easy to verify that $L_{x} y=x \circ y$ for any $x, y \in \mathbb{R}^{n}$. Recall that each $x=\left(x_{1}, x_{2}\right) \in \mathbb{R} \times \mathbb{R}^{n-1}$ admits a spectral factorization, associated with $\mathscr{K}^{n}$, of the form

$$
x=\lambda_{1}(x) u_{x}^{(1)}+\lambda_{2}(x) u_{x}^{(2)}
$$

where $\lambda_{1}(x), \lambda_{2}(x) \in \mathbb{R}$ and $u_{x}^{(1)}, u_{x}^{(2)} \in \mathbb{R}^{n}$ are the spectral values and the associated spectral vectors of $x$, respectively, with respect to the Jordan product, defined by

$$
\begin{gathered}
\lambda_{i}(x):=x_{1}+(-1)^{i}\left\|x_{2}\right\|, \\
u_{x}^{(i)}:=\frac{1}{2}\left(\begin{array}{c}
1 \\
(-1)^{i} \tilde{x}_{2}
\end{array}\right), \quad \text { for } i=1,2,
\end{gathered}
$$

with $\tilde{x}_{2}=x_{2} /\left\|x_{2}\right\|$ if $x_{2} \neq 0$ and otherwise being any vector in $\mathbb{R}^{n-1}$ satisfying $\left\|\tilde{x}_{2}\right\|=1$.

Definition 2. The determinant of a vector $x \in \mathbb{R}^{n}$ is defined as $\operatorname{det}(x):=\lambda_{1}(x) \lambda_{2}(x)$, and a vector $x$ is said to be invertible if its determinant $\operatorname{det}(x)$ is nonzero.

By the formula of spectral factorization, it is easy to compute that the projection of $x \in \mathbb{R}^{n}$ onto the closed convex cone $\mathscr{K}^{n}$, denoted by $\Pi_{\mathscr{K}^{n}}(x)$, has the expression

$$
\Pi_{\mathscr{K}^{n}}(x)=\max \left(0, \lambda_{1}(x)\right) u_{x}^{(1)}+\max \left(0, \lambda_{2}(x)\right) u_{x}^{(2)} .
$$

Define $|x|:=2 \Pi_{\mathscr{K}^{n}}(x)-x$. Then, using the expression of $\Pi_{\mathscr{K}^{n}}(x)$, it follows that

$$
|x|=\left|\lambda_{1}(x)\right| u_{x}^{(1)}+\left|\lambda_{2}(x)\right| u_{x}^{(2)}
$$

The spectral factorization of the vectors $x, x^{2}, \sqrt{x}$ and the matrix $L_{x}$ have various interesting properties (see [13]). We list several properties that we will use later.

Property 3. For any $x=\left(x_{1}, x_{2}\right) \in \mathbb{R} \times \mathbb{R}^{n-1}$ with spectral factorization (15), we have the following.

(a) $x^{2}=\lambda_{1}^{2}(x) u_{x}^{(1)}+\lambda_{2}^{2}(x) u_{x}^{(2)} \in \mathscr{K}^{n}$.

(b) If $x \in \mathscr{K}^{n}$, then $0 \leq \lambda_{1}(x) \leq \lambda_{2}(x)$ and $\sqrt{x}=$ $\sqrt{\lambda_{1}(x)} u_{x}^{(1)}+\sqrt{\lambda_{2}(x)} u_{x}^{(2)}$.

(c) If $x \in \operatorname{int} \mathscr{K}^{n}$, then $0<\lambda_{1}(x) \leq \lambda_{2}(x)$ and $L_{x}$ is invertible with

$$
L_{x}^{-1}=\frac{1}{\operatorname{det}(x)}\left[\begin{array}{cc}
x_{1} & -x_{2}^{T} \\
-x_{2} & \frac{\operatorname{det}(x)}{x_{1}} I+\frac{x_{2} x_{2}^{T}}{x_{1}}
\end{array}\right] .
$$

(d) $L_{x} \geq 0$ (resp., $L_{x}>0$ ) if and only if $x \in \mathscr{K}^{n}$ (resp., $\left.x \in \operatorname{int} \mathscr{K}^{n}\right)$.
The following lemma states a result for the arrow matrices associated with $x, y \in \mathbb{R}^{n}$ and $z \geq_{\mathscr{K}^{n}} \sqrt{x^{2}+y^{2}}$, which will be used in the next section to characterize an important property for the elements of Clarke's Jacobian of $\phi_{\mathrm{FB}}$ at a general point.

Lemma 4. For any given $x, y \in \mathbb{R}^{n}$ and $z \succ_{\mathscr{K}^{n}} 0$, if $z^{2} \succeq_{\mathscr{K}^{n}} x^{2}+$ $y^{2}$, then

$$
\left\|\left[L_{z}^{-1} L_{x} L_{z}^{-1} L_{y}\right]\right\|_{2} \leq 1
$$

where $\|A\|_{2}$ means the spectral norm of a real matrix $A$. Consequently, it holds that

$\left\|L_{z}^{-1} L_{x} \Delta u+L_{z}^{-1} L_{y} \Delta v\right\| \leq \sqrt{\|\Delta u\|^{2}+\|\Delta v\|^{2}}, \quad \forall \Delta u, \Delta v \in \mathbb{R}^{n}$.

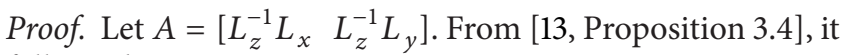
follows that

$$
A A^{T}=L_{z}^{-1}\left(L_{x}^{2}+L_{y}^{2}\right) L_{z}^{-1} \preceq L_{z}^{-1} L_{z}^{2} L_{z}^{-1}=I .
$$

This shows that $\|A\|_{2} \leq 1$, and the first part follows. Note that, for any $\xi \in \mathbb{R}^{2 n}$,

$$
\|A \xi\|^{2}=\xi^{T} A^{T} A \xi \leq \lambda_{\max }\left(A^{T} A\right)\|\xi\|^{2} \leq\|\xi\|^{2} .
$$

By letting $\xi=(\Delta u, \Delta v) \in \mathbb{R}^{n} \times \mathbb{R}^{n}$, we immediately obtain the second part.

The following two lemmas state the properties of $x, y$ with $x^{2}+y^{2} \in \mathbf{b d} \mathscr{K}^{n}$ which are often used in the subsequent sections. The proof of Lemma 5 is given in [3, Lemma 2].

Lemma 5. For any $x=\left(x_{1}, x_{2}\right), y=\left(y_{1}, y_{2}\right) \in \mathbb{R} \times \mathbb{R}^{n-1}$ with $x^{2}+y^{2} \in \mathbf{b d} \mathscr{K}^{n}$, one has

$$
\begin{gathered}
x_{1}^{2}=\left\|x_{2}\right\|^{2}, \quad y_{1}^{2}=\left\|y_{2}\right\|^{2}, \\
x_{1} y_{1}=x_{2}^{T} y_{2}, \quad x_{1} y_{2}=y_{1} x_{2} .
\end{gathered}
$$

Lemma 6. For any $x=\left(x_{1}, x_{2}\right), y=\left(y_{1}, y_{2}\right) \in \mathbb{R} \times \mathbb{R}^{n-1}$, let $w=\left(w_{1}, w_{2}\right):=x^{2}+y^{2}$.

(a) If $w \in \mathbf{b d} \mathscr{K}^{n}$, then for any $g=\left(g_{1}, g_{2}\right), h=\left(h_{1}, h_{2}\right) \in$ $\mathbb{R} \times \mathbb{R}^{n-1}$, it holds that

$$
\begin{gathered}
\left(x_{1} x_{2}+y_{1} y_{2}\right)^{T}\left(x_{1} g_{2}+g_{1} x_{2}+y_{1} h_{2}+h_{1} y_{2}\right) \\
=\left(x_{1}^{2}+y_{1}^{2}\right)\left(x^{T} g+y^{T} h\right) .
\end{gathered}
$$

(b) If $w \in \mathbf{b d}^{+} \mathscr{K}^{n}$, then the following four equalities hold

$$
\begin{array}{ll}
\frac{x_{1} w_{2}}{\left\|w_{2}\right\|}=x_{2}, & \frac{x_{2}^{T} w_{2}}{\left\|w_{2}\right\|}=x_{1}, \\
\frac{y_{1} w_{2}}{\left\|w_{2}\right\|}=y_{2}, & \frac{y_{2}^{T} w_{2}}{\left\|w_{2}\right\|}=y_{1} ;
\end{array}
$$


and consequently the expression of $\phi_{\mathrm{FB}}(x, y)$ can be simplified as

$$
\phi_{\mathrm{FB}}(x, y)=\left(\begin{array}{c}
x_{1}+y_{1}-\sqrt{x_{1}^{2}+y_{1}^{2}} \\
x_{2}+y_{2}-\frac{x_{1} x_{2}+y_{1} y_{2}}{\sqrt{x_{1}^{2}+y_{1}^{2}}}
\end{array}\right) .
$$

Proof. (a) The result is direct by the equalities of Lemma 5 since $x^{2}+y^{2} \in \mathbf{b d} \mathscr{K}^{n}$.

(b) Since $w \in \mathbf{b d}^{+} \mathscr{K}^{n}$, we must have $w_{2}=2\left(x_{1} x_{2}+\right.$ $\left.y_{1} y_{2}\right) \neq 0$. Using Lemma $5, w_{2}=2\left(x_{1} x_{2}+y_{1} y_{2}\right)$ and $\left\|w_{2}\right\|=$ $w_{1}=2\left(x_{1}^{2}+y_{1}^{2}\right)$, we easily obtain the first part. Note that $\phi_{\mathrm{FB}}(x, y)=(x+y)-\sqrt{w}$. Using Property 3(b) and Lemma 5 yields (27).

When $x, y \in \mathbf{b d} \mathscr{K}^{n}$ satisfies the complementary condition, we have the following result.

Lemma 7. For any given $x=\left(x_{1}, x_{2}\right), y=\left(y_{1}, y_{2}\right) \in \mathbb{R} \times$ $\mathbb{R}^{n-1}$, if $x, y \in \mathbf{b d} \mathscr{K}^{n}$ and $\langle x, y\rangle=0$, then there exists $a$ constant $\alpha>0$ such that $x_{1}=\alpha y_{1}$ and $x_{2}=-\alpha y_{2}$.

Proof. Since $x, y \in \mathbf{b d} \mathscr{K}^{n}$, we have that $x_{1}=\left\|x_{2}\right\|$ and $y_{1}=$ $\left\|y_{2}\right\|$, and consequently,

$$
0=\langle x, y\rangle=x_{1} y_{1}+x_{2}^{T} y_{2}=\left\|x_{2}\right\|\left\|y_{2}\right\|+x_{2}^{T} y_{2} .
$$

This means that there exists $\alpha>0$ such that $x_{2}=-\alpha y_{2}$, and then $x_{1}=\alpha y_{1}$.

Next we recall from [14] the strong regularity for a solution of generalized equation

$$
0 \in \phi(z)+\mathcal{N}_{D}(z)
$$

where $\phi$ is a continuously differentiable mapping from a finite dimensional real vector space $\mathbb{Z}$ to itself, $D$ is a closed convex set in $\mathbb{Z}$, and $\mathcal{N}_{D}(z)$ is the normal cone of $D$ at $z$. As will be shown in Section 4, the KKT condition (7) can be written in the form of (29).

Definition 8 . We say that $\bar{z}$ is a strongly regular solution of the generalized equation (29) if there exist neighborhood $\mathscr{B}$ of the origin $0 \in \mathbb{Z}$ and $\mathscr{V}$ of $\bar{z}$ such that for every $\delta \in \mathscr{B}$, the linearized generalized equation $\delta \in \phi(\bar{z})+\mathscr{J}_{z} \phi(\bar{z})(z-\bar{z})+$ $\mathcal{N}_{D}(z)$ has a unique solution in $\mathscr{V}$, denoted by $z_{\mathscr{V}}(\delta)$, and the mapping $z_{\mathscr{V}}: \mathscr{B} \rightarrow \mathscr{V}$ is Lipschitz continuous.

To close this section, we recall from [15] Clarke's (generalized) Jacobian of a locally Lipschitz mapping. Let $S \subset$ $\mathbb{R}^{n}$ be an open set and $\Xi: S \rightarrow \mathbb{R}^{n}$ a locally Lipschitz continuous function on $S$. By Rademacher's theorem, $\Xi$ is almost everywhere $F$ (réchet)-differentiable in $S$. We denote by $S_{\Xi}$ the set of points in $S$ where $\Xi$ is $F$-differentiable. Then Clarke's Jacobian of $\Xi$ at $y$ is defined by $\partial \Xi(y):=$ $\operatorname{conv}\left\{\partial_{B} \Xi(y)\right\}$, where "conv" means the convex hull, and $B$ subdifferential $\partial_{B} \Xi(y)$, a name coined in [16], has the form

$$
\partial_{B} \Xi(y):=\left\{V: V=\lim _{k \rightarrow \infty} \mathscr{J}_{y} \Xi\left(y^{k}\right), y^{k} \longrightarrow y, y^{k} \in S_{\Xi}\right\} .
$$

For the concept of (strong) semismoothness, please refer to the literature $[5,6]$.

Unless otherwise stated, in the rest of this paper, for any $x \in \mathbb{R}^{n}(n>1)$, we write $x=\left(x_{1}, x_{2}\right)$, where $x_{1}$ is the first component of $x$ and $x_{2}$ is a column vector consisting of the remaining $n-1$ entries of $x$. For any $x=\left(x_{1}, x_{2}\right), y=$ $\left(y_{1}, y_{2}\right) \in \mathbb{R} \times \mathbb{R}^{n-1}$, let

$$
\begin{array}{r}
w=w(x, y):=x^{2}+y^{2}, \quad \widetilde{w}_{2}:=\frac{w_{2}}{\left\|w_{2}\right\|}, \\
\quad \text { if } w_{2} \neq 0, \quad z=z(x, y)=\sqrt{w(x, y) .}
\end{array}
$$

\section{Directional Derivative and $B$-Subdifferential}

The function $\phi_{\mathrm{FB}}$ is directionally differentiable everywhere by [2, Corollary 3.3]. But, to the best of our knowledge, the expression of its directional derivative is not given in the literature. In this section, we derive its expression and then prove that the $B$-subdifferential of $\phi_{\mathrm{FB}}$ at a general point coincides with that of its directional derivative function at the origin. Throughout this section, we assume that $\mathscr{K}=\mathscr{K}^{n}$.

Proposition 9. For any given $x=\left(x_{1}, x_{2}\right), y=\left(y_{1}, y_{2}\right) \in$ $\mathbb{R} \times \mathbb{R}^{n-1}$, the directional derivative $\phi_{\mathrm{FB}}^{\prime}((x, y) ;(g, h))$ of $\phi_{\mathrm{FB}}$ at $(x, y)$ with the direction $(g, h)$ has the following form.

(a) If $(x, y)=(0,0)$, then $\phi_{\mathrm{FB}}^{\prime}((x, y) ;(g, h))=\phi_{\mathrm{FB}}(g, h)$.

(b) If $x^{2}+y^{2} \in \operatorname{int} \mathscr{K}^{n}$, then $\phi_{\mathrm{FB}}^{\prime}((x, y) ;(g, h))=(I-$ $\left.L_{z}^{-1} L_{x}\right) g+\left(I-L_{z}^{-1} L_{y}\right) h$.

(c) If $x^{2}+y^{2} \in \mathbf{b d}^{+} \mathscr{K}^{n}$, then

$$
\begin{aligned}
\phi_{\mathrm{FB}}^{\prime}((x, y) ;(g, h)) & \\
= & (g+h)-\frac{\varphi(g, h)}{2}\left(\begin{array}{c}
1 \\
-\widetilde{w}_{2}
\end{array}\right)+\frac{x_{2}^{T} g_{2}+y_{2}^{T} h_{2}}{2 \sqrt{x_{1}^{2}+y_{1}^{2}}}\left(\begin{array}{c}
0 \\
\widetilde{w}_{2}
\end{array}\right) \\
& -\frac{1}{2 \sqrt{x_{1}^{2}+y_{1}^{2}}}\left(\begin{array}{c}
x^{T} g+y^{T} h \\
2 x_{1} g_{2}+g_{1} x_{2}+2 y_{1} h_{2}+h_{1} y_{2}
\end{array}\right),
\end{aligned}
$$

where $g=\left(g_{1}, g_{2}\right), h=\left(h_{1}, h_{2}\right) \in \mathbb{R} \times \mathbb{R}^{n-1}$, and $\varphi: \mathbb{R}^{n} \times \mathbb{R}^{n} \rightarrow \mathbb{R}$ is defined by

$\varphi(g, h)$

$$
:=\frac{\sqrt{\left(x_{1} g_{1}-x_{2}^{T} g_{2}+y_{1} h_{1}-y_{2}^{T} h_{2}\right)^{2}+\left\|x_{1} h_{2}-h_{1} x_{2}+g_{1} y_{2}-y_{1} g_{2}\right\|^{2}}}{\sqrt{x_{1}^{2}+y_{1}^{2}}} .
$$

Proof. Part (a) is immediate by noting that $\phi_{\mathrm{FB}}$ is a positively homogeneous function. Part (b) is due to [13, Proposition 5.2]. We next prove part (c) by two subcases as shown in the following. In the rest of proof, we let $\lambda_{1}, \lambda_{2}$ with $\lambda_{1} \leq \lambda_{2}$ 
denote the spectral values of $w$. Since $w=x^{2}+y^{2} \in \mathbf{b d}^{+} \mathscr{K}^{n}$, we have $w_{2} \neq 0$, and from Lemma 6 (b) it follows that

$$
\begin{gathered}
w_{1}=\left\|w_{2}\right\|=2\left\|x_{1} x_{2}+y_{1} y_{2}\right\| \\
=2\left\|x_{1}^{2} \widetilde{w}_{2}+y_{1}^{2} \widetilde{w}_{2}\right\|=2\left(x_{1}^{2}+y_{1}^{2}\right), \\
\lambda_{1}=w_{1}-\left\|w_{2}\right\|=0, \quad \lambda_{2}=w_{1}+\left\|w_{2}\right\|=4\left(x_{1}^{2}+y_{1}^{2}\right) .
\end{gathered}
$$

(c.1): $(x+t g)^{2}+(y+t h)^{2} \in \mathbf{b d}^{+} \mathscr{K}^{n}$ for sufficiently small $t>0$. In this case, from Lemma $6(\mathrm{~b})$, we know that $\phi_{\mathrm{FB}}(x+$ $\operatorname{tg}, y+t h)$ has the following expression:

$$
\left(\begin{array}{c}
\left(x_{1}+y_{1}\right)+t\left(g_{1}+h_{1}\right)-\sqrt{\left(x_{1}+t g_{1}\right)^{2}+\left(y_{1}+t h_{1}\right)^{2}} \\
\left(x_{2}+y_{2}\right)+t\left(g_{2}+h_{2}\right) \\
-\frac{\left(x_{1}+t g_{1}\right)\left(x_{2}+t g_{2}\right)+\left(y_{1}+t h_{1}\right)\left(y_{2}+t h_{2}\right)}{\sqrt{\left(x_{1}+t g_{1}\right)^{2}+\left(y_{1}+t h_{1}\right)^{2}}}
\end{array}\right) .
$$

Let $\left[\phi_{\mathrm{FB}}(x, y)\right]_{1}$ be the first element of $\phi_{\mathrm{FB}}(x, y)$ and $\left[\phi_{\mathrm{FB}}(x, y)\right]_{2}$ the vector consisting of the rest $n-1$ components of $\phi_{\mathrm{FB}}(x, y)$. By the above expression of $\phi_{\mathrm{FB}}(x+t g, y+t h)$,

$$
\begin{aligned}
\lim _{t \downarrow 0} \frac{\left[\phi_{\mathrm{FB}}(x+t g, y+t h)\right]_{1}-\left[\phi_{\mathrm{FB}}(x, y)\right]_{1}}{t} \\
\quad=\left(g_{1}+h_{1}\right)-\lim _{t \downarrow 0} \frac{\sqrt{\left(x_{1}+t g_{1}\right)^{2}+\left(y_{1}+t h_{1}\right)^{2}}-\sqrt{x_{1}^{2}+y_{1}^{2}}}{t} \\
\quad=\left(g_{1}+h_{1}\right)-\frac{x_{1} g_{1}+y_{1} h_{1}}{\sqrt{x_{1}^{2}+y_{1}^{2}}},
\end{aligned}
$$$$
\lim _{t \downarrow 0} \frac{\left[\phi_{\mathrm{FB}}(x+t g, y+t h)\right]_{2}-\left[\phi_{\mathrm{FB}}(x, y)\right]_{2}}{t}
$$$$
=\left(g_{2}+h_{2}\right)
$$$$
-\lim _{t \downarrow 0}\left[\frac{\left(x_{1}+t g_{1}\right)\left(x_{2}+t g_{2}\right)+\left(y_{1}+t h_{1}\right)\left(y_{2}+t h_{2}\right)}{t \sqrt{\left(x_{1}+t g_{1}\right)^{2}+\left(y_{1}+t h_{1}\right)^{2}}}\right.
$$$$
\left.-\frac{x_{1} x_{2}+y_{1} y_{2}}{t \sqrt{x_{1}^{2}+y_{1}^{2}}}\right]
$$

$$
\begin{aligned}
= & \left(g_{2}+h_{2}\right)-\frac{g_{1} x_{2}+x_{1} g_{2}+y_{1} h_{2}+h_{1} y_{2}}{\sqrt{x_{1}^{2}+y_{1}^{2}}} \\
& -\lim _{t \downarrow 0}\left[\frac{x_{1} x_{2}+y_{1} y_{2}}{t \sqrt{\left(x_{1}+t g_{1}\right)^{2}+\left(y_{1}+t h_{1}\right)^{2}}}-\frac{x_{1} x_{2}+y_{1} y_{2}}{t \sqrt{x_{1}^{2}+y_{1}^{2}}}\right] \\
= & \left(g_{2}+h_{2}\right)-\frac{g_{1} x_{2}+x_{1} g_{2}+y_{1} h_{2}+h_{1} y_{2}}{\sqrt{x_{1}^{2}+y_{1}^{2}}} \\
& +\frac{\left(x_{1} x_{2}+y_{1} y_{2}\right)\left(x_{1} g_{1}+y_{1} h_{1}\right)}{\left(x_{1}^{2}+y_{1}^{2}\right) \sqrt{x_{1}^{2}+y_{1}^{2}}} \\
= & \left(g_{2}+h_{2}\right)-\frac{x_{1} g_{2}+y_{1} h_{2}}{\sqrt{x_{1}^{2}+y_{1}^{2}}},
\end{aligned}
$$

where the last equality is using $x_{1} y_{2}=y_{1} x_{2}$ by Lemma 5 . The above two limits imply

$$
\phi_{\mathrm{FB}}^{\prime}((x, y) ;(g, h))=(g+h)-\frac{x_{1}}{\sqrt{x_{1}^{2}+y_{1}^{2}}} g-\frac{y_{1}}{\sqrt{x_{1}^{2}+y_{1}^{2}}} h .
$$

(c.2): $(x+t g)^{2}+(y+t h)^{2} \in$ int $\mathscr{K}^{n}$ for sufficiently small $t>0$. Let $u=\left(u_{1}, u_{2}\right):=(x+t g)^{2}+(y+t h)^{2}$ with the spectral values $\mu_{1}, \mu_{2}$. An elementary calculation gives

$$
\begin{aligned}
u_{1}= & \|x+t g\|^{2}+\|y+t h\|^{2}=w_{1}+2 t\left(x^{T} g+y^{T} h\right) \\
& +t^{2}\left(\|g\|^{2}+\|h\|^{2}\right), \\
u_{2}= & 2\left(x_{1}+t g_{1}\right)\left(x_{2}+t g_{2}\right)+2\left(y_{1}+t h_{1}\right)\left(y_{2}+t h_{2}\right) \\
= & w_{2}+2 t\left(x_{1} g_{2}+g_{1} x_{2}+y_{1} h_{2}+h_{1} y_{2}\right) \\
& +2 t^{2}\left(g_{1} g_{2}+h_{1} h_{2}\right) .
\end{aligned}
$$

Also, since $w_{2} \neq 0$, applying the Taylor formula of $\|\cdot\|$ at $w_{2}$ and Lemma 6(a) yields

$$
\begin{aligned}
\left\|u_{2}\right\| & =\left\|w_{2}\right\|+\frac{w_{2}^{T}\left(u_{2}-w_{2}\right)}{\left\|w_{2}\right\|}+o(t) \\
& =\left\|w_{2}\right\|+2 t\left(x^{T} g+y^{T} h\right)+o(t) .
\end{aligned}
$$

Now using the definition of $\phi_{\mathrm{FB}}$ and noting that $\lambda_{1}=0$ and $w_{2} \neq 0$, we have that

$$
\begin{aligned}
\phi_{\mathrm{FB}}(x+t g, y+t h)-\phi_{\mathrm{FB}}(x, y) \\
=(x+t g+y+t h)-\sqrt{u}-(x+y)+\sqrt{w} \\
=t(g+h)-\left(\begin{array}{c}
\frac{\sqrt{\mu_{1}}+\sqrt{\mu_{2}}-\sqrt{\lambda_{2}}}{2} \\
\frac{\sqrt{\mu_{2}}-\sqrt{\mu_{1}}}{2} \frac{u_{2}}{\left\|u_{2}\right\|}-\frac{\sqrt{\lambda_{2}}}{2} \frac{w_{2}}{\left\|w_{2}\right\|}
\end{array}\right),
\end{aligned}
$$


which in turn implies that

$$
\begin{aligned}
& \phi_{\mathrm{FB}}^{\prime}((x, y) ;(g, h)) \\
& =(g+h)-\left(\begin{array}{c}
\lim _{t \downarrow 0} \frac{\sqrt{\mu_{1}}+\sqrt{\mu_{2}}-\sqrt{\lambda_{2}}}{2 t} \\
\lim _{t \downarrow 0}\left(\frac{\sqrt{\mu_{2}}-\sqrt{\mu_{1}}}{2 t} \frac{u_{2}}{\left\|u_{2}\right\|}-\frac{\sqrt{\lambda_{2}}}{2 t} \frac{w_{2}}{\left\|w_{2}\right\|}\right)
\end{array}\right) .
\end{aligned}
$$

We first calculate $\lim _{t \downarrow 0}\left(\left(\sqrt{\mu_{2}}-\sqrt{\lambda_{2}}\right) / t\right)$. Using (38) and (40), it is easy to see that

$$
\begin{aligned}
\mu_{2}-\lambda_{2} & =\left(u_{1}-w_{1}\right)+\left(\left\|u_{2}\right\|-\left\|w_{2}\right\|\right) \\
& =4 t\left(x^{T} g+y^{T} h\right)+o(t)
\end{aligned}
$$

and consequently,

$$
\begin{aligned}
\lim _{t \downarrow 0} \frac{\sqrt{\mu_{2}}-\sqrt{\lambda_{2}}}{t} & =\lim _{t \downarrow 0} \frac{\mu_{2}-\lambda_{2}}{t} \cdot \frac{1}{\sqrt{\mu_{2}}+\sqrt{\lambda_{2}}} \\
& =\frac{x^{T} g+y^{T} h}{2 \sqrt{\lambda_{2}}}=\frac{x^{T} g+y^{T} h}{\sqrt{x_{1}^{2}+y_{1}^{2}}} .
\end{aligned}
$$

We next calculate $\lim _{t \downarrow 0}\left(\sqrt{\mu_{1}} / t\right)$. Since $w_{1}-\left\|w_{2}\right\|=0$, using (38)-(39) and Lemma 6(a),

$$
\begin{aligned}
& \mu_{1}=\left(u_{1}-w_{1}\right)-\left(\left\|u_{2}\right\|-\left\|w_{2}\right\|\right) \\
& =\left(u_{1}-w_{1}\right)-\frac{\left\|u_{2}\right\|^{2}-\left\|w_{2}\right\|^{2}}{\left\|u_{2}\right\|+\left\|w_{2}\right\|} \\
& =2 t\left(x^{T} g+y^{T} h\right)-4 t \frac{w_{2}^{T}\left(x_{1} g_{2}+g_{1} x_{2}+y_{1} h_{2}+h_{1} y_{2}\right)}{\left\|u_{2}\right\|+\left\|w_{2}\right\|} \\
& +t^{2}\left(\|g\|^{2}+\|h\|^{2}\right) \\
& -4 t^{2} \frac{\left\|g_{1} x_{2}+x_{1} g_{2}+y_{1} h_{2}+h_{1} y_{2}\right\|^{2}}{\left\|u_{2}\right\|+\left\|w_{2}\right\|} \\
& -4 t^{2} \frac{w_{2}^{T}\left(g_{1} g_{2}+h_{1} h_{2}\right)}{\left\|u_{2}\right\|+\left\|w_{2}\right\|}+o\left(t^{2}\right) \\
& =2 t\left(x^{T} g+y^{T} h\right)-8 t \frac{\left(x_{1}^{2}+y_{1}^{2}\right)\left(x^{T} g+y^{T} h\right)}{\left\|u_{2}\right\|+\left\|w_{2}\right\|} \\
& +t^{2}\left(\|g\|^{2}+\|h\|^{2}\right)+o\left(t^{2}\right) \\
& -4 t^{2} \frac{\left\|g_{1} x_{2}+x_{1} g_{2}+y_{1} h_{2}+h_{1} y_{2}\right\|^{2}}{\left\|u_{2}\right\|+\left\|w_{2}\right\|} \\
& -8 t^{2} \frac{\left(x_{1} x_{2}+y_{1} y_{2}\right)^{T}\left(g_{1} g_{2}+h_{1} h_{2}\right)}{\left\|u_{2}\right\|+\left\|w_{2}\right\|}
\end{aligned}
$$

Using $\left\|w_{2}\right\|=2\left(x_{1}^{2}+y_{1}^{2}\right)$ and (40), we simplify the sum of the first two terms in (45) as

$$
\begin{gathered}
2 t\left(x^{T} g+y^{T} h\right)-\frac{4 t\left\|w_{2}\right\|\left(x^{T} g+y^{T} h\right)}{\left\|u_{2}\right\|+\left\|w_{2}\right\|} \\
=2 t\left(x^{T} g+y^{T} h\right) \frac{\left\|u_{2}\right\|-\left\|w_{2}\right\|}{\left\|u_{2}\right\|+\left\|w_{2}\right\|} \\
=\frac{4 t^{2}\left(x^{T} g+y^{T} h\right)^{2}}{\left\|u_{2}\right\|+\left\|w_{2}\right\|}+o\left(t^{2}\right) .
\end{gathered}
$$

Then, from (45) and $\left\|w_{2}\right\|=2\left(x_{1}^{2}+y_{1}^{2}\right)$, we obtain that

$$
\begin{aligned}
& \lim _{t \downarrow 0} \frac{\mu_{1}}{t^{2}} \\
& =\frac{\left(x_{1}^{2}+y_{1}^{2}\right)\left(\|g\|^{2}+\|h\|^{2}\right)-\left\|g_{1} x_{2}+x_{1} g_{2}+y_{1} h_{2}+h_{1} y_{2}\right\|^{2}}{x_{1}^{2}+y_{1}^{2}} \\
& +\frac{\left(x^{T} g+y^{T} h\right)^{2}-2\left(x_{1} x_{2}+y_{1} y_{2}\right)^{T}\left(g_{1} g_{2}+h_{1} h_{2}\right)}{x_{1}^{2}+y_{1}^{2}} .
\end{aligned}
$$

We next make simplification for the numerator of the right hand side of (47). Note that

$$
\begin{aligned}
&\left(x_{1}^{2}+y_{1}^{2}\right)\left(\|g\|^{2}+\|h\|^{2}\right)-\left\|g_{1} x_{2}+x_{1} g_{2}+y_{1} h_{2}+h_{1} y_{2}\right\|^{2} \\
&=\left(x_{1}^{2}+y_{1}^{2}\right)\left(\|g\|^{2}+\|h\|^{2}\right)-\left\|g_{1} x_{2}+x_{1} g_{2}\right\|^{2} \\
&-\left\|y_{1} h_{2}+h_{1} y_{2}\right\|^{2}-2\left(g_{1} x_{2}+x_{1} g_{2}\right)^{T}\left(y_{1} h_{2}+h_{1} y_{2}\right) \\
&=x_{1}^{2}\|h\|^{2}+y_{1}^{2}\|g\|^{2}-2 x_{1} g_{1} x_{2}^{T} g_{2}-2 y_{1} h_{1} y_{2}^{T} h_{2} \\
&-2\left(g_{1} x_{2}+x_{1} g_{2}\right)^{T}\left(y_{1} h_{2}+h_{1} y_{2}\right), \\
&\left(x^{T} g+\right.\left.y^{T} h\right)^{2}-2\left(x_{1} x_{2}+y_{1} y_{2}\right)^{T}\left(g_{1} g_{2}+h_{1} h_{2}\right) \\
&=\left(x_{1} g_{1}+x_{2}^{T} g_{2}\right)^{2}+\left(y_{1} h_{1}+y_{2}^{T} h_{2}\right)^{2}+2 x^{T} g y^{T} h \\
&-2\left(x_{1} x_{2}+y_{1} y_{2}\right)^{T}\left(g_{1} g_{2}+h_{1} h_{2}\right) \\
&=\left(x_{1} g_{1}\right)^{2}+\left(x_{2}^{T} g_{2}\right)^{2}+\left(y_{1} h_{1}\right)^{2}+\left(y_{2}^{T} h_{2}\right)^{2} \\
&+2 x^{T} g y^{T} h-2 x_{1} h_{1} x_{2}^{T} h_{2}-2 g_{1} y_{1} g_{2}^{T} y_{2} .
\end{aligned}
$$


Therefore, adding the last two equalities and using Lemma 5 yield that

$$
\begin{aligned}
\left(x_{1}^{2}+y_{1}^{2}\right) & \left(\|g\|^{2}+\|h\|^{2}\right)-\left\|g_{1} x_{2}+x_{1} g_{2}+y_{1} h_{2}+h_{1} y_{2}\right\|^{2} \\
& +\left(x^{T} g+y^{T} h\right)^{2}-2\left(x_{1} x_{2}+y_{1} y_{2}\right)^{T}\left(g_{1} g_{2}+h_{1} h_{2}\right) \\
= & \left(x_{1}^{2}\|h\|^{2}-2 x_{1} h_{1} x_{2}^{T} h_{2}\right)+\left(y_{1}^{2}\|g\|^{2}-2 g_{1} y_{1} g_{2}^{T} y_{2}\right) \\
& +\left(\left(x_{1} g_{1}\right)^{2}+\left(x_{2}^{T} g_{2}\right)^{2}-2 x_{1} g_{1} x_{2}^{T} g_{2}\right) \\
& +\left(\left(y_{1} h_{1}\right)^{2}+\left(y_{2}^{T} h_{2}\right)^{2}-2 y_{1} h_{1} y_{2}^{T} h_{2}\right)+2 x^{T} g y^{T} h \\
& -2\left(g_{1} x_{2}+x_{1} g_{2}\right)^{T}\left(y_{1} h_{2}+h_{1} y_{2}\right) \\
= & \left\|x_{1} h_{2}-h_{1} x_{2}\right\|^{2}+\left\|g_{1} y_{2}-y_{1} g_{2}\right\|^{2}+\left(x_{1} g_{1}-x_{2}^{T} g_{2}\right)^{2} \\
& +\left(y_{1} h_{1}-y_{2}^{T} h_{2}\right)^{2}+2\left(g_{1} x_{1}+g_{2}^{T} x_{2}\right)\left(y_{1} h_{1}+y_{2}^{T} h_{2}\right) \\
& -2\left(g_{1} x_{2}+x_{1} g_{2}\right)^{T}\left(y_{1} h_{2}+h_{1} y_{2}\right) \\
= & \left\|x_{1} h_{2}-h_{1} x_{2}\right\|^{2}+\left\|g_{1} y_{2}-y_{1} g_{2}\right\|^{2}+\left(x_{1} g_{1}-x_{2}^{T} g_{2}\right)^{2} \\
& +\left(y_{1} h_{1}-y_{2}^{T} h_{2}\right)^{2}+2\left(x_{1} h_{2}-h_{1} x_{2}\right)^{T}\left(g_{1} y_{2}-g_{2} y_{1}\right) \\
& +2\left(x_{1} g_{1}-x_{2}^{T} g_{2}\right)\left(y_{1} h_{1}-y_{2}^{T} h_{2}\right) \\
= & \left\|x_{1} h_{2}-h_{1} x_{2}+g_{1} y_{2}-y_{1} g_{2}\right\|^{2} \\
& +\left(x_{1} g_{1}-x_{2}^{T} g_{2}+y_{1} h_{1}-y_{2}^{T} h_{2}\right)^{2} .
\end{aligned}
$$

Combining this equality with (47) and using the definition of $\varphi$ in (33), we readily get

$$
\lim _{t \downarrow 0} \frac{\sqrt{\mu_{1}}}{t}=\varphi(g, h) .
$$

We next calculate $\lim _{t \downarrow 0}\left[\left(\left(\sqrt{\mu_{2}}-\sqrt{\mu_{1}}\right) / 2 t\right)\left(u_{2} /\left\|u_{2}\right\|\right)-\right.$ $\left.\left(\left(\sqrt{\lambda_{2}} / 2 t\right)\left(w_{2} /\left\|w_{2}\right\|\right)\right)\right]$. To this end, we also need to take a look at $\left\|w_{2}\right\| u_{2}-\left\|u_{2}\right\| w_{2}$. From (38)-(39) and (40), it follows that

$$
\begin{aligned}
\left\|w_{2}\right\| u_{2}-\left\|u_{2}\right\| & w_{2} \\
=2 t\left\|w_{2}\right\|[ & \left(x_{1} g_{2}+g_{1} x_{2}+y_{1} h_{2}+h_{1} y_{2}\right) \\
& \left.-\left(x^{T} g+y^{T} h\right) \widetilde{w}_{2}\right]+o(t) .
\end{aligned}
$$

Together with (44) and (50), we have that

$$
\begin{aligned}
\lim _{t \downarrow 0}[ & \left.\frac{\sqrt{\mu_{2}}-\sqrt{\mu_{1}}}{2 t} \frac{u_{2}}{\left\|u_{2}\right\|}-\frac{\sqrt{\lambda_{2}}}{2 t} \frac{w_{2}}{\left\|w_{2}\right\|}\right] \\
= & -\lim _{t \downarrow 0} \frac{\sqrt{\mu_{1}}}{2 t} \frac{u_{2}}{\left\|u_{2}\right\|}+\lim _{t \downarrow 0}\left[\frac{\sqrt{\mu_{2}}}{2 t} \frac{u_{2}}{\left\|u_{2}\right\|}-\frac{\sqrt{\lambda_{2}}}{2 t} \frac{w_{2}}{\left\|w_{2}\right\|}\right] \\
= & -\lim _{t \downarrow 0} \frac{\sqrt{\mu_{1}}}{2 t} \frac{u_{2}}{\left\|u_{2}\right\|}+\lim _{t \downarrow 0} \frac{\sqrt{\mu_{2}}-\sqrt{\lambda_{2}}}{2 t} \frac{u_{2}}{\left\|u_{2}\right\|} \\
& +\lim _{t \downarrow 0} \frac{\sqrt{\lambda_{2}}\left(\left\|w_{2}\right\| u_{2}-\left\|u_{2}\right\| w_{2}\right)}{2 t\left\|u_{2}\right\|\left\|w_{2}\right\|} \\
= & -\frac{\varphi(g, h)}{2} \widetilde{w}_{2}+\frac{x_{1} g_{2}+g_{1} x_{2}+y_{1} h_{2}+h_{1} y_{2}}{\sqrt{x_{1}^{2}+y_{1}^{2}}} \\
& -\frac{x^{T} g+y^{T} h}{2 \sqrt{x_{1}^{2}+y_{1}^{2}}} \widetilde{w}_{2} \\
= & -\frac{\varphi(g, h)}{2} \widetilde{w}_{2}+\frac{2 x_{1} g_{2}+g_{1} x_{2}+2 y_{1} h_{2}+h_{1} y_{2}}{2 \sqrt{x_{1}^{2}+y_{1}^{2}}} \\
& -\frac{x_{2}^{T} g_{2}+y_{2}^{T} h_{2}}{2 \sqrt{x_{1}^{2}+y_{1}^{2}}} \widetilde{w}_{2},
\end{aligned}
$$

where the last equality is using $x_{1} \widetilde{w}_{2}=x_{2}$ and $y_{1} \widetilde{w}_{2}=y_{2}$. Combining with (42), (44), and (50), a suitable rearrangement shows that $\phi_{\mathrm{FB}}^{\prime}((x, y) ;(g, h))$ has the expression (32).

Finally, we show that, when $(x+t g)^{2}+(y+t h)^{2} \in \mathbf{b d}^{+} \mathscr{K}^{n}$ for sufficiently small $t>0$, the formula in (32) reduces to the one in (37). Indeed, an elementary calculation yields

$$
\begin{aligned}
& \lambda_{1}\left((x+t g)^{2}+(y+t h)^{2}\right) \\
&=\left[\|x+t g\|^{2}+\|y+t h\|^{2}\right]^{2} \\
& \quad-4\left\|\left(x_{1}+t g_{1}\right)\left(x_{2}+t g_{2}\right)+\left(y_{1}+t h_{1}\right)\left(y_{2}+t h_{2}\right)\right\|^{2} \\
&=4 t^{2} \varphi(g, h) \sqrt{x_{1}^{2}+y_{1}^{2}} \\
& \quad+4 t^{3}\left(x^{T} g+y^{T} h\right)\left(\|g\|^{2}+\|h\|^{2}\right) \\
& \quad-8 t^{2}\left(x_{1} g_{2}+g_{1} x_{2}+y_{1} h_{2}+h_{1} y_{2}\right)^{T}\left(g_{1} g_{2}+h_{1} h_{2}\right) \\
&+t^{4}\left[\left(\|g\|^{2}+\|h\|^{2}\right)^{2}-2\left\|g_{1} g_{2}+h_{1} h_{2}\right\|^{2}\right] \\
&= 4 t^{2} \varphi(g, h) \sqrt{x_{1}^{2}+y_{1}^{2}}+o\left(t^{2}\right) .
\end{aligned}
$$

This implies that if $(x+t g)^{2}+(y+t h)^{2} \in \mathbf{b d}^{+} \mathscr{K}^{n}$ for sufficiently small $t>0$, that is, $\lambda_{1}\left((x+t g)^{2}+(y+t h)^{2}\right)=0$ for sufficiently small $t>0$, then $\varphi(g, h)=0$, and hence $x_{1} g_{1}+y_{1} h_{1}-\left(x_{2}^{T} g_{2}+\right.$ 
$\left.y_{2}^{T} h_{2}\right)=0$. Thus, $\phi_{\mathrm{FB}}^{\prime}((x, y) ;(g, h))$ in $(32)$ can be simplified as

$$
\begin{aligned}
& (g+h)+\frac{x_{1} g_{1}+y_{1} h_{1}}{2 \sqrt{x_{1}^{2}+y_{1}^{2}}}\left(\begin{array}{c}
0 \\
\widetilde{w}_{2}
\end{array}\right)-\frac{1}{2 \sqrt{x_{1}^{2}+y_{1}^{2}}} \\
& \times\left(\begin{array}{c}
2 x_{1} g_{1}+2 y_{1} h_{1} \\
2 x_{1} g_{2}+g_{1} x_{2}+2 y_{1} h_{2}+h_{1} y_{2}
\end{array}\right) \\
& =(g+h)-\frac{x_{1}}{\sqrt{x_{1}^{2}+y_{1}^{2}}} g-\frac{y_{1}}{\sqrt{x_{1}^{2}+y_{1}^{2}}} h,
\end{aligned}
$$

where the equality is using $g_{1} x_{2}=g_{1} x_{1} \widetilde{w}_{2}, h_{1} y_{2}=h_{1} y_{1} \widetilde{w}_{2}$. The proof is complete.

As a consequence of Proposition 9, we have the following sufficient and necessary characterizations for the (continuously) differentiable points of $\phi_{\mathrm{FB}}$ and $\vartheta(x):=|x|$.

Corollary 10. (a) The function $\phi_{\mathrm{FB}}$ is (continuously) differentiable at $(x, y) \in \mathbb{R}^{n} \times \mathbb{R}^{n}$ if and only if $z(x, y) \in$ int $\mathscr{K}^{n}$, where $z(x, y)$ is defined in (31). Also, when $z(x, y) \in$ int $\mathscr{K}^{n}$, one has

$$
\mathscr{J} \phi_{\mathrm{FB}}(x, y)=\left[I-L_{z(x, y)}^{-1} L_{x} I-L_{z(x, y)}^{-1} L_{y}\right] .
$$

(b) The function $\vartheta(x):=|x|$ is (continuously) differentiable at $x \in \mathbb{R}^{n}$ if and only if $x$ is invertible. Also, when $x$ is invertible, $\mathscr{J} \mathcal{V}(x)=L_{|x|}^{-1} L_{x}$.

Proof. (a) The "if" part is direct by [13, Proposition 5.2]. We next prove the "only if" part. If $\phi_{\mathrm{FB}}$ is differentiable at $(x, y)$, then $\phi_{\mathrm{FB}}^{\prime}((x, y) ;(g, h))$ is a linear function of $(g, h)$, which by Proposition 9 holds only if $z(x, y) \in$ int $\mathscr{K}^{n}$. The formula of $\mathscr{J} \phi_{\mathrm{FB}}(x, y)$ is given in [13].

(b) Since $\vartheta(x)=\sqrt{x^{2}}$, by part (a) $\vartheta$ is (continuously) differentiable at $x \in \mathbb{R}^{n}$ if and only if $|x| \in$ int $\mathscr{K}^{n}$, which is equivalent to requiring that $x$ is invertible since $|x| \in \mathscr{K}^{n}$ always holds. When $x$ is invertible, the formula of $\mathscr{J} \vartheta(x)$ follows from part (a).

For any given $x=\left(x_{1}, x_{2}\right), y=\left(y_{1}, y_{2}\right) \in \mathbb{R} \times \mathbb{R}^{n-1}$ with $x^{2}+y^{2} \in \mathbf{b d}^{+} \mathscr{K}^{n}$, define

$$
\begin{gathered}
\theta_{1}(g, h):=x_{1} g_{1}-x_{2}^{T} g_{2}+y_{1} h_{1}-y_{2}^{T} h_{2}, \\
\theta_{2}(g, h):=x_{1} h_{2}-h_{1} x_{2}+g_{1} y_{2}-y_{1} g_{2}
\end{gathered}
$$

for any $g=\left(g_{1}, g_{2}\right), h=\left(h_{1}, h_{2}\right) \in \mathbb{R} \times \mathbb{R}^{n-1}$, and let $\theta(g, h):=$ $\left(\theta_{1}(g, h), \theta_{2}(g, h)\right)$. Then, comparing with (33), we can rewrite the function $\varphi$ as

$$
\varphi(g, h)=\frac{\|\theta(g, h)\|}{\sqrt{x_{1}^{2}+y_{1}^{2}}}, \quad \forall g, h \in \mathbb{R}^{n} .
$$

Note that the Euclidean norm $\|\cdot\|$ is globally Lipschitz continuous and strongly semismooth everywhere in $\mathbb{R}^{n}$, and $\theta(\cdot, \cdot)$ is a linear function. Then, (57) implies that $\varphi$ is globally Lipschitz continuous and strongly semismooth everywhere in $\mathbb{R}^{n} \times \mathbb{R}^{n}$ by [17, Theorem 19]. Also, the function $\varphi$ is differentiable at $(g, h)$ if and only if $\varphi(g, h)>0$. The following lemma characterizes the $B$-subdifferential of the function $\varphi$ at the origin.

Lemma 11. For any given $(x, y)$ with $x^{2}+y^{2} \in \mathbf{b d}^{+} \mathscr{K}^{n}$, let $\varphi$ be defined by (33). Then, the B-subdifferential of the function $\varphi$ at $(0,0)$ takes the following form:

$$
\begin{aligned}
& \partial_{B} \varphi(0,0)=\left\{\left[\left(\frac{\xi_{2}^{T} y_{2}+\xi_{1} x_{1}}{\sqrt{x_{1}^{2}+y_{1}^{2}}}, \frac{-y_{1} \xi_{2}^{T}-\xi_{1} x_{2}^{T}}{\sqrt{x_{1}^{2}+y_{1}^{2}}}\right),\right.\right. \\
& \left.\left(\frac{-\xi_{2}^{T} x_{2}+\xi_{1} y_{1}}{\sqrt{x_{1}^{2}+y_{1}^{2}}}, \frac{x_{1} \xi_{2}^{T}-\xi_{1} y_{2}^{T}}{\sqrt{x_{1}^{2}+y_{1}^{2}}}\right)\right]: \\
& \left.\left(\xi_{1}, \xi_{2}\right) \in \mathbb{R} \times \mathbb{R}^{n-1} \text { satisfies } \xi_{1}^{2}+\left\|\xi_{2}\right\|^{2}=1\right\} \text {. }
\end{aligned}
$$

Proof. Let $\left(u^{T}, v^{T}\right) \in \partial_{B} \varphi(0,0)$. By the definition of the elements in $\partial_{B} \varphi(0,0)$, there exists a sequence $\left\{\left(g^{k}, h^{k}\right)\right\}$ in $\mathbb{R}^{n} \times$ $\mathbb{R}^{n}$ converging to $(0,0)$ with $\varphi\left(g^{k}, h^{k}\right)>0$ such that

$$
\left(u^{T}, v^{T}\right)=\lim _{k \rightarrow \infty} \mathscr{J} \varphi\left(g^{k}, h^{k}\right) .
$$

By (57), a simple computation shows that such $\left(u^{T}, v^{T}\right)$ belongs to the set on the right hand side of (58). Thus, $\partial_{B} \varphi(0,0)$ is included in the set on the right hand side of (58). In fact, $\xi_{1}$ and $\xi_{2}$ in (58) are the limit points of $\left\{\theta_{1}\left(g^{k}, h^{k}\right) /\left\|\theta\left(g^{k}, h^{k}\right)\right\|\right\}$ and $\left\{\theta_{2}\left(g^{k}, h^{k}\right) /\left\|\theta\left(g^{k}, h^{k}\right)\right\|\right\}$, respectively.

Conversely, let $\left(u^{T}, v^{T}\right)$ be an arbitrary element of the set on the right hand side of (58). Then, there exists a $\left(\xi_{1}, \xi_{2}\right) \in$ $\mathbb{R} \times \mathbb{R}^{n-1}$ with $\xi_{1}^{2}+\left\|\xi_{2}\right\|^{2}=1$ such that

$$
\begin{aligned}
& u=\left(\frac{\xi_{2}^{T} y_{2}+\xi_{1} x_{1}}{\sqrt{x_{1}^{2}+y_{1}^{2}}}, \frac{-y_{1} \xi_{2}-\xi_{1} x_{2}}{\sqrt{x_{1}^{2}+y_{1}^{2}}}\right), \\
& v=\left(\frac{-\xi_{2}^{T} x_{2}+\xi_{1} y_{1}}{\sqrt{x_{1}^{2}+y_{1}^{2}}}, \frac{x_{1} \xi_{2}-\xi_{1} y_{2}}{\sqrt{x_{1}^{2}+y_{1}^{2}}}\right) .
\end{aligned}
$$

Take the sequence $\left\{\left(g^{k}, h^{k}\right)\right\} \subset \mathbb{R}^{n} \times \mathbb{R}^{n}$ with $g^{k}=\left(x_{1} \xi_{1} /\right.$ $\left.k,-y_{1} \xi_{2} / k\right)$ and $h^{k}=\left(y_{1} \xi_{1} / k, x_{1} \xi_{2} / k\right)$. Clearly, $\left(g^{k}, h^{k}\right) \rightarrow$ $(0,0)$ as $k \rightarrow \infty$. Also, by Lemma 5 , it is easy to verify that

$$
\begin{aligned}
& \theta_{1}\left(g^{k}, h^{k}\right)=\frac{1}{k}\left(x_{1}^{2}+y_{1}^{2}\right) \xi_{1}, \\
& \theta_{2}\left(g^{k}, h^{k}\right)=\frac{1}{k}\left(x_{1}^{2}+y_{1}^{2}\right) \xi_{2} .
\end{aligned}
$$

This shows that $\varphi\left(g^{k}, h^{k}\right)>0$ and $\mathscr{J} \varphi\left(g^{k}, h^{k}\right)=\left(u^{T}, v^{T}\right)$. Hence, $\left(u^{T}, v^{T}\right) \in \partial_{B} \varphi(0,0)$. Thus, the set on the right hand side of (58) is included in $\partial_{B} \varphi(0,0)$. 
Now we may prove the equivalence between the $B$ subdifferential of $\phi_{\mathrm{FB}}$ at a general point $(x, y)$ and that of its directional derivative function $\phi_{\mathrm{FB}}^{\prime}((x, y) ;(\cdot, \cdot))$ at $(0,0)$. This result corresponds to that of $[18$, Lemma 14$]$ established for the NR SOC function $\phi_{\mathrm{NR}}$.

Lemma 12. For any given $(x, y) \in \mathbb{R}^{n} \times \mathbb{R}^{n}$, let $\psi_{\mathrm{FB}}(\cdot, \cdot) \equiv$ $\phi_{\mathrm{FB}}^{\prime}((x, y) ;(\cdot, \cdot))$. Then,

$$
\partial_{B} \phi_{\mathrm{FB}}(x, y)=\partial_{B} \psi_{\mathrm{FB}}(0,0) .
$$

Proof. The result is direct by Proposition 9(a)-(b) and Lemma A.1 in the Appendix.

Using Lemma 12, we may present an upper estimation for Clarke's Jacobian of $\phi_{\mathrm{FB}}$ at the point $(x, y)$ with $x^{2}+y^{2} \epsilon$ bd $^{+} \mathscr{K}^{n}$, which will be used in the next section.

Proposition 13. For any given $x=\left(x_{1}, x_{2}\right), y=\left(y_{1}, y_{2}\right)$ with $x^{2}+y^{2} \in \mathbf{b d}^{+} \mathscr{K}^{n}$, one has

$$
\begin{aligned}
& \partial \phi_{\mathrm{FB}}(x, y) \\
& \quad \text { for } u=\left(u_{1}, u_{2}\right), \\
& \quad v=\left(v_{1}, v_{2}\right) \text { satisfying the inequalities } \\
& \left|u_{1}\right| \leq\left\|u_{2}\right\| \leq 1,\left|v_{1}\right| \leq\left\|v_{2}\right\| \leq 1, \\
& \left(u_{1}-v_{1}\right) \leq\left\|u_{2}-v_{2}\right\|,\left(u_{1}+v_{1}\right) \leq\left\|u_{2}+v_{2}\right\|, \\
& \left(u_{1}-v_{1}\right)^{2}+\left\|u_{2}+v_{2}\right\|^{2} \leq 2, \\
& \left(u_{1}+v_{1}\right)^{2}+\left\|u_{2}-v_{2}\right\|^{2} \leq 2, \\
& \left(1, \widetilde{w}_{2}^{T}\right) u=0,\left(1, \widetilde{w}_{2}^{T}\right) v=0, \\
& \left.\left(1,-\widetilde{w}_{2}^{T}\right) u=2 u_{1},\left(1,-\widetilde{w}_{2}^{T}\right) v=2 v_{1}\right\}
\end{aligned}
$$

where $X$ and $Y$ are $n \times n$ real symmetric matrices defined as follows:

$$
\begin{aligned}
& X=\frac{1}{\sqrt{2 w_{1}}}\left(\begin{array}{cc}
x_{1} & x_{2}^{T} \\
x_{2} & 2 x_{1} I-\widetilde{w}_{2} x_{2}^{T}
\end{array}\right), \\
& Y=\frac{1}{\sqrt{2 w_{1}}}\left(\begin{array}{cc}
y_{1} & y_{2}^{T} \\
y_{2} & 2 y_{1} I-\widetilde{w}_{2} y_{2}^{T}
\end{array}\right) .
\end{aligned}
$$

Proof. We first make simplifications for the last two terms in (32) by $X, Y$. Note that

$$
\begin{aligned}
& \frac{x_{2}^{T} g_{2}+y_{2}^{T} h_{2}}{2 \sqrt{x_{1}^{2}+y_{1}^{2}}}\left(\begin{array}{c}
0 \\
\widetilde{w}_{2}
\end{array}\right)-\frac{1}{2 \sqrt{x_{1}^{2}+y_{1}^{2}}} \\
& \quad \times\left(\begin{array}{c}
x^{T} g+y^{T} h \\
2 x_{1} g_{2}+g_{1} x_{2}+2 y_{1} h_{2}+h_{1} y_{2}
\end{array}\right)
\end{aligned}
$$

$$
\begin{aligned}
= & \frac{1}{2 \sqrt{x_{1}^{2}+y_{1}^{2}}}\left(\begin{array}{c}
0 \\
\widetilde{w}_{2}
\end{array}\right)\left(\begin{array}{ll}
0 & x_{2}^{T}
\end{array}\right) g \\
& +\frac{1}{2 \sqrt{x_{1}^{2}+y_{1}^{2}}}\left(\begin{array}{c}
0 \\
\widetilde{w}_{2}
\end{array}\right)\left(\begin{array}{ll}
0 & y_{2}^{T}
\end{array}\right) h \\
& -\frac{1}{2 \sqrt{x_{1}^{2}+y_{1}^{2}}}\left(\begin{array}{cc}
x_{1} & x_{2}^{T} \\
x_{2} & 2 x_{1} I
\end{array}\right) g \\
& -\frac{1}{2 \sqrt{x_{1}^{2}+y_{1}^{2}}}\left(\begin{array}{cc}
y_{1} & y_{2}^{T} \\
y_{2} & 2 y_{1} I
\end{array}\right) h \\
= & -X g-Y h,
\end{aligned}
$$

where the last equality is using $w_{1}=2\left(x_{1}^{2}+y_{1}^{2}\right)$. Therefore, from (32), we have

$$
\phi_{\mathrm{FB}}^{\prime}((x, y) ;(g, h))=(g+h)-\frac{\varphi(g, h)}{2}\left(\begin{array}{c}
1 \\
-\widetilde{w}_{2}
\end{array}\right)-X g-Y h,
$$

$\forall g, h \in \mathbb{R}^{n}$

Now, applying Lemma 12, we immediately obtain that

$$
\begin{aligned}
& \partial \phi_{\mathrm{FB}}(x, y) \\
& =\left\{\left[I-X-\frac{1}{2}\left(\begin{array}{c}
1 \\
-\widetilde{w}_{2}
\end{array}\right) u^{T} I-Y-\frac{1}{2}\left(\begin{array}{c}
1 \\
-\widetilde{w}_{2}
\end{array}\right) v^{T}\right] \mid\right. \\
& \quad \text { for } u=\left(u_{1}, u_{2}\right), v=\left(v_{1}, v_{2}\right) \\
& \left.\quad \text { with }\left(u^{T}, v^{T}\right) \in \partial \varphi(0,0)\right\},
\end{aligned}
$$

where, by Lemma 11 and the definition of Clarke's Jacobian,

$$
\begin{aligned}
\partial \varphi(0,0)=\{ & {\left[\left(\frac{\xi_{2}^{T} y_{2}+\xi_{1} x_{1}}{\sqrt{x_{1}^{2}+y_{1}^{2}}}, \frac{-y_{1} \xi_{2}^{T}-\xi_{1} x_{2}^{T}}{\sqrt{x_{1}^{2}+y_{1}^{2}}}\right),\right.} \\
& \left.\left(\frac{-\xi_{2}^{T} x_{2}+\xi_{1} y_{1}}{\sqrt{x_{1}^{2}+y_{1}^{2}}}, \frac{x_{1} \xi_{2}^{T}-\xi_{1} y_{2}^{T}}{\sqrt{x_{1}^{2}+y_{1}^{2}}}\right)\right]:
\end{aligned}
$$$$
\left.\left(\xi_{1}, \xi_{2}\right) \in \mathbb{R} \times \mathbb{R}^{n-1} \text { satisfies } \xi_{1}^{2}+\left\|\xi_{2}\right\|^{2} \leq 1\right\} \text {. }
$$

Let $\left(u^{T}, v^{T}\right) \in \partial \varphi(0,0)$ with $u=\left(u_{1}, u_{2}\right), v=\left(v_{1}, v_{2}\right) \epsilon$ $\mathbb{R} \times \mathbb{R}^{n-1}$. Then, it suffices to prove that such $u$ and $v$ satisfy 
all inequalities and equalities in (63). By (68), there exists a $\operatorname{vector}\left(\xi_{1}, \xi_{2}\right) \in \mathbb{R} \times \mathbb{R}^{n-1}$ with $\xi_{1}^{2}+\left\|\xi_{2}\right\|^{2} \leq 1$ such that

$$
\begin{array}{ll}
u_{1}=\frac{\xi_{2}^{T} y_{2}+\xi_{1} x_{1}}{\sqrt{x_{1}^{2}+y_{1}^{2}}}, & u_{2}=\frac{-y_{1} \xi_{2}-\xi_{1} x_{2}}{\sqrt{x_{1}^{2}+y_{1}^{2}}}, \\
v_{1}=\frac{-\xi_{2}^{T} x_{2}+\xi_{1} y_{1}}{\sqrt{x_{1}^{2}+y_{1}^{2}}}, & v_{2}=\frac{x_{1} \xi_{2}-\xi_{1} y_{2}}{\sqrt{x_{1}^{2}+y_{1}^{2}}} .
\end{array}
$$

Using Lemma 5, it is immediate to obtain that

$$
\begin{aligned}
\left|\xi_{2}^{T} y_{2}+\xi_{1} x_{1}\right|^{2} & =\left(\xi_{2}^{T} y_{2}\right)^{2}+\left(\xi_{1} x_{1}\right)^{2}+2 \xi_{1} x_{1} \xi_{2}^{T} y_{2} \\
& \leq\left\|\xi_{2}\right\|^{2} y_{1}^{2}+\xi_{1}^{2} x_{1}^{2}+2 \xi_{1} y_{1} \xi_{2}^{T} x_{2} \\
& =\left\|-y_{1} \xi_{2}-\xi_{1} x_{2}\right\|^{2} \\
& \leq\left\|\xi_{2}\right\|^{2} y_{1}^{2}+\xi_{1}^{2} x_{1}^{2}+\left\|\xi_{2}\right\|^{2} x_{1}^{2}+\xi_{1}^{2}\left\|y_{2}\right\|^{2} \\
& =\left(\left\|\xi_{2}\right\|^{2}+\xi_{1}^{2}\right)\left(x_{1}^{2}+y_{1}^{2}\right) \leq x_{1}^{2}+y_{1}^{2} .
\end{aligned}
$$

This means that $\left|u_{1}\right| \leq\left\|u_{2}\right\| \leq 1$. Similarly, we also have $\left|v_{1}\right| \leq$ $\left\|v_{2}\right\| \leq 1$. We next prove that $\left(u_{1}-v_{1}\right)^{2}-\left\|u_{2}-v_{2}\right\|^{2} \leq 0$. By Lemma 5, it is easy to verify that

$$
\begin{aligned}
\left(x_{2}-y_{2}\right)\left(x_{1}+y_{1}\right) & =\left(x_{2}+y_{2}\right)\left(x_{1}-y_{1}\right), \\
\left(x_{1}-y_{1}\right)^{2} & =\left\|x_{2}-y_{2}\right\|^{2} .
\end{aligned}
$$

Using the two equalities, it is not hard to calculate that

$$
\begin{aligned}
\left(u_{1}-v_{1}\right)^{2}-\left\|u_{2}-v_{2}\right\|^{2} & \\
= & \frac{\left(\xi_{2}^{T} y_{2}+\xi_{1} x_{1}+\xi_{2}^{T} x_{2}-\xi_{1} y_{1}\right)^{2}}{x_{1}^{2}+y_{1}^{2}} \\
& -\frac{\left\|-y_{1} \xi_{2}-\xi_{1} x_{2}-x_{1} \xi_{2}+\xi_{1} y_{2}\right\|^{2}}{x_{1}^{2}+y_{1}^{2}} \\
= & \frac{\left(\xi_{2}^{T} y_{2}+\xi_{2}^{T} x_{2}\right)^{2}-\left\|\xi_{2}\right\|^{2}\left(y_{1}+x_{1}\right)^{2}}{x_{1}^{2}+y_{1}^{2}} \leq 0 .
\end{aligned}
$$

Similarly, we also have $\left(u_{1}+v_{1}\right)^{2} \leq\left\|u_{2}+v_{2}\right\|^{2}$. In addition, we have that

$$
\begin{aligned}
\left(u_{1}-v_{1}\right)^{2}+\left\|u_{2}+v_{2}\right\|^{2} & \\
= & \frac{\left(\xi_{2}^{T} y_{2}+\xi_{1} x_{1}+\xi_{2}^{T} x_{2}-\xi_{1} y_{1}\right)^{2}}{x_{1}^{2}+y_{1}^{2}} \\
& +\frac{\left\|-y_{1} \xi_{2}-\xi_{1} x_{2}+x_{1} \xi_{2}-\xi_{1} y_{2}\right\|^{2}}{x_{1}^{2}+y_{1}^{2}} \\
\leq & \frac{\left(\xi_{1}^{2}+\left\|\xi_{2}\right\|^{2}\right)\left[\left(y_{1}-x_{1}\right)^{2}+\left\|x_{2}+y_{2}\right\|^{2}\right]}{x_{1}^{2}+y_{1}^{2}} \\
= & \frac{2\left(\xi_{1}^{2}+\left\|\xi_{2}\right\|^{2}\right)\left(x_{1}^{2}+y_{1}^{2}\right)}{x_{1}^{2}+y_{1}^{2}} \leq 2 .
\end{aligned}
$$

A similar argument also yields $\left(u_{1}+v_{1}\right)^{2}+\left\|u_{2}-v_{2}\right\|^{2} \leq 2$. The last four equalities in (63) are direct by Lemma 5 and the expression of $u_{1}, u_{2}, v_{1}$, and $v_{2}$.

To close this section, we establish a relation between the $B$-subdifferential of $\phi_{\mathrm{FB}}$ at a complementarity point pair and that of $\phi_{\mathrm{NR}}$ at the corresponding point pair.

Lemma 14. Let $(\bar{x}, \bar{y}) \in \mathbb{R}^{n} \times \mathbb{R}^{n}$ satisfy $\bar{x} \in \mathscr{K}^{n}, \bar{y} \in \mathscr{K}^{n}$ and $\langle\bar{x}, \bar{y}\rangle=0$. Then,

$$
\partial_{B} \phi_{\mathrm{NR}}(\bar{x}, \bar{y}) \subseteq \partial_{B} \phi_{\mathrm{FB}}(\bar{x}, \bar{y})
$$

Proof. Since $(\bar{x}, \bar{y})$ satisfies $\bar{x} \in \mathscr{K}^{n}, \bar{y} \in \mathscr{K}^{n}$ and $\langle\bar{x}, \bar{y}\rangle=0$, there exist spectral vectors $u^{(1)}, u^{(2)} \in \mathbb{R}^{n}$ and nonnegative real numbers $\lambda_{1}, \lambda_{2}$ and $v_{1}, v_{2}$ such that

$$
\bar{x}=\lambda_{1} u^{(1)}+\lambda_{2} u^{(2)}, \quad \bar{y}=v_{1} u^{(1)}+v_{2} u^{(2)} .
$$

Indeed, if $\bar{x}=0$ or $\bar{y}=0$, then the statement clearly holds. If $\bar{x} \neq 0$ and $\bar{y} \neq 0$, then the condition that $\bar{x} \in \mathscr{K}^{n}, \bar{y} \in \mathscr{K}^{n}$, and $\langle\bar{x}, \bar{y}\rangle=0$ implies $\bar{x}, \bar{y} \in \mathbf{b d} \mathscr{K}^{n}$. From Lemma 7, there exists $\alpha>0$ such that $\bar{x}_{2}=-\alpha \bar{y}_{2}$ and $\bar{x}_{1}=\alpha \bar{y}_{1}$. Together with the spectral factorizations of $\bar{x}$ and $\bar{y}$, the conclusion in (75) follows. Since $\left\langle u^{(1)}, u^{(2)}\right\rangle=0$ and $\left\|u^{(1)}\right\|=\left\|u^{(2)}\right\|=1 / \sqrt{2}$, using (75) and $\langle\bar{x}, \bar{y}\rangle=0$ yields that $\lambda_{1} v_{1}=\lambda_{2} v_{2}=0$. This, along with the nonnegativity of $\lambda_{1}, \lambda_{2}$ and $\nu_{1}, v_{2}$, implies

$$
\bar{x}=\frac{|\bar{x}-\bar{y}|+(\bar{x}-\bar{y})}{2}, \quad \bar{y}=\frac{|\bar{x}-\bar{y}|-(\bar{x}-\bar{y})}{2} .
$$

By the definition of $\phi_{\mathrm{NR}}$, we have $\phi_{\mathrm{NR}}(x, y)=(x+y)-\psi(x, y)$ for $x, y \in \mathbb{R}^{n}$, where

$$
\psi(x, y):=\frac{1}{2}[(x+y)+|x-y|] \quad \forall x, y \in \mathbb{R}^{n}
$$

Comparing with the definition of $\phi_{\mathrm{FB}}$, we only need to prove the following inclusion:

$$
\partial_{B} \psi(\bar{x}, \bar{y}) \subseteq \partial_{B} z(\bar{x}, \bar{y})
$$

For this purpose, let $\left[\begin{array}{ll}U & V\end{array}\right] \in \partial_{B} \psi(\bar{x}, \bar{y})$. By the definition of the elements in $\partial_{B} \psi(\bar{x}, \bar{y})$ and Corollary $10(\mathrm{~b})$, there exists a sequence $\left\{\left(x^{k}, y^{k}\right)\right\} \subset \mathbb{R}^{n} \times \mathbb{R}^{n}$ converging to $(\bar{x}, \bar{y})$ with $c^{k} \equiv x^{k}-y^{k}$ invertible such that

$$
\begin{aligned}
{\left[\begin{array}{ll}
U & V
\end{array}\right] } & =\lim _{k \rightarrow \infty} \mathscr{J} \psi\left(x^{k}, y^{k}\right) \\
& =\lim _{k \rightarrow \infty}\left[\frac{1}{2}\left(I+L_{\left|c^{k}\right|}^{-1} L_{c^{k}}\right) \frac{1}{2}\left(I-L_{\left|c^{k}\right|}^{-1} L_{c^{k}}\right)\right] .
\end{aligned}
$$

For each $k$, let $\widehat{x}^{k}=\left(\left|c^{k}\right|+c^{k}\right) / 2$ and $\widehat{y}^{k}=\left(\left|c^{k}\right|-c^{k}\right) /$ 2. Then, using $c^{k}=x^{k}-y^{k}$ and (76), we have that $\widehat{x}^{k} \rightarrow \bar{x}$ and $\widehat{y}^{k} \rightarrow \bar{y}$ as $k \rightarrow \infty$. Also, $z\left(\hat{x}^{k}, \widehat{y}^{k}\right)=\sqrt{\left(c^{k}\right)^{2}}=$ 
$\left|c^{k}\right| \succ_{\mathscr{K}^{n}} 0$. By Corollary 10(a), the function $z(\cdot, \cdot)$ is continuously differentiable at $\left(\hat{x}^{k}, \widehat{y}^{k}\right)$ with

$$
\begin{aligned}
\mathscr{J} z\left(\hat{x}^{k}, \hat{y}^{k}\right) & =\left[\begin{array}{ll}
L_{z\left(\hat{x}^{k}, \hat{y}^{k}\right)}^{-1} L_{\hat{x}^{k}} & L_{z\left(\hat{x}^{k}, \hat{y}^{k}\right)}^{-1} L_{\hat{y}^{k}}
\end{array}\right] \\
& =\left[\begin{array}{ll}
L_{\left|c^{k}\right|}^{-1} L_{\left(\left|c^{k}\right|+c^{k}\right) / 2} & L_{\left|c^{k}\right|}^{-1} L_{\left(\left|c^{k}\right|-c^{k}\right) / 2}
\end{array}\right] .
\end{aligned}
$$

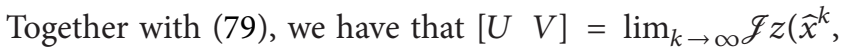
$\left.\hat{y}^{k}\right)$. This shows that $\left[\begin{array}{ll}U & V\end{array}\right] \partial_{B} z(\bar{x}, \bar{y})$, and the inclusion in (78) follows. The proof is complete.

\section{Nonsingularity Conditions}

This section studies the nonsingularity of Clarke's Jacobian of $E_{\mathrm{FB}}$ at a KKT point. Let $(\bar{\zeta}, \bar{x}, \bar{\mu}, \bar{s}, \bar{y}) \in \mathbb{R}^{n} \times \mathscr{K} \times \mathbb{R}^{m} \times \mathbb{R}^{n} \times \mathscr{K}$ be a KKT point of the SOCP (4), that is,

$$
\begin{gathered}
\mathscr{J}_{\zeta, x} L(\bar{\zeta}, \bar{x}, \bar{\mu}, \bar{s}, \bar{y})=0, \quad h(\bar{\zeta})=0, \\
g(\bar{\zeta})-\bar{x}=0, \quad-\bar{y} \in \mathcal{N}_{\mathscr{K}}(\bar{x}) .
\end{gathered}
$$

Taking into account that $-\bar{y} \in \mathcal{N}_{\mathscr{K}}(\bar{x})$ if and only if $\bar{x}$ and $\bar{y}$ satisfy

$$
\bar{x}_{j} \in \mathscr{K}^{n_{j}}, \quad \bar{y}_{j} \in \mathscr{K}^{n_{j}}, \quad\left\langle\bar{x}_{j}, \bar{y}_{j}\right\rangle=0, \quad j=1,2, \ldots, r,
$$

we introduce the following index sets associated with $\bar{x}$ and $\bar{y}$ :

$$
\begin{gathered}
J_{I 0}:=\left\{j \mid \bar{x}_{j} \in \text { int } \mathscr{K}^{n_{j}}, \bar{y}_{j}=0\right\}, \\
J_{0 I}:=\left\{j \mid \bar{x}_{j}=0, \bar{y}_{j} \in \text { int } \mathscr{K}^{n_{j}}\right\}, \\
J_{B B}:=\left\{j \mid \bar{x}_{j}, \bar{y}_{j} \in \mathbf{b} \mathbf{d}^{+} \mathscr{K}^{n_{j}}\right\}, \\
J_{B 0}:=\left\{j \mid \bar{x}_{j} \in \mathbf{b} \mathbf{d}^{+} \mathscr{K}^{n_{j}}, \bar{y}_{j}=0\right\}, \\
J_{0 B}:=\left\{j \mid \bar{x}_{j}=0, \bar{y}_{j} \in \mathbf{b d} \mathbf{d}^{+} \mathscr{K}^{n_{j}}\right\}, \\
J_{00}:=\left\{j \mid \bar{x}_{j}=0, \bar{y}_{j}=0\right\} .
\end{gathered}
$$

From [19], we learn that the above six index sets form a partition of $\{1,2, \ldots, r\}$.

First of all, let us take a careful look at the properties of the elements in $\partial \phi_{\mathrm{FB}}\left(\bar{x}_{j}, \bar{y}_{j}\right)$ for $j \in J_{B 0} \cup J_{0 B}$, as stated in the following. The proof of Lemma 15 is given in the Appendix.

Lemma 15. For $(\bar{x}, \bar{y})$ satisfying (82), let $\left[U_{j} V_{j}\right] \in \partial \phi_{\mathrm{FB}}\left(\bar{x}_{j}\right.$, $\bar{y}_{j}$ ) for each $j$. Then,

(a) when $j \in J_{B 0}$, there exists an $n_{j} \times n_{j}$ orthogonal matrix $Q_{j}=\left[\begin{array}{lll}q_{j} & \widehat{Q}_{j} & q_{j}^{\prime}\end{array}\right]$ with

$$
q_{j}=\frac{1}{\sqrt{2}}\left(\begin{array}{c}
1 \\
\tilde{x}_{j 2}
\end{array}\right), \quad q_{j}^{\prime}=\frac{1}{\sqrt{2}}\left(\begin{array}{c}
1 \\
-\tilde{x}_{j 2}
\end{array}\right), \quad \tilde{x}_{j 2}=\frac{\bar{x}_{j 2}}{\left\|\bar{x}_{j 2}\right\|}
$$

such that $U_{j}=Q_{j} D_{j} Q_{j}^{T}, V_{j}=Q_{j} \Lambda_{j} Q_{j}^{T}$, where $D_{j}$ and $\Lambda_{j}$ take one of the forms

$$
\begin{gathered}
D_{j}=0, \quad \Lambda_{j}=I, \quad Q_{j}=I ; \\
D_{j}=\operatorname{diag}(0,0, \ldots, 0,1), \quad \Lambda_{j}=\operatorname{diag}(1,1, \ldots, 1,0) ; \\
D_{j}=\operatorname{diag}\left(0,0, \ldots, 0,1-u_{j 1}\right), \\
\Lambda_{j}=\left(\begin{array}{ccc}
1 & 0 & 0 \\
0 & I & 0 \\
0 & -\frac{1}{\sqrt{2}} v_{j}^{T} \widehat{Q}_{j} & 1-v_{j 1}
\end{array}\right)
\end{gathered}
$$

with $u_{j}=\left(u_{j 1}, u_{j 2}\right), v_{j}=\left(v_{j 1}, v_{j 2}\right) \in \mathbb{R} \times \mathbb{R}^{n_{j}-1}$ satisfying $u_{j 1}, v_{j 1}<1$,

(b) when $j \in J_{0 B}$, there exists an $n_{j} \times n_{j}$ orthogonal matrix $Q_{j}=\left[\begin{array}{lll}q_{j} & \widehat{Q}_{j} & q_{j}^{\prime}\end{array}\right]$ with

$$
q_{j}=\frac{1}{\sqrt{2}}\left(\begin{array}{c}
1 \\
\tilde{y}_{j 2}
\end{array}\right), \quad q_{j}^{\prime}=\frac{1}{\sqrt{2}}\left(\begin{array}{c}
1 \\
-\tilde{y}_{j 2}
\end{array}\right), \quad \tilde{y}_{j 2}=\frac{\bar{y}_{j 2}}{\left\|\bar{y}_{j 2}\right\|}
$$

such that $U_{j}=Q_{j} D_{j} Q_{j}^{T}, V_{j}=Q_{j} \Lambda_{j} Q_{j}^{T}$, where $D_{j}$ and $\Lambda_{j}$ take one of the forms

$$
\begin{gathered}
D_{j}=I, \quad \Lambda_{j}=0, \quad Q_{j}=I ; \\
D_{j}=\operatorname{diag}(1,1, \ldots, 1,0), \quad \Lambda_{j}=\operatorname{diag}(0,0, \ldots, 0,1) ; \\
D_{j}=\left(\begin{array}{ccc}
1 & 0 & 0 \\
0 & I & 0 \\
0 & -\frac{1}{\sqrt{2}} u_{j}^{T} \widehat{Q}_{j} & 1-u_{j 1}
\end{array}\right), \\
\Lambda_{j}=\operatorname{diag}\left(0,0, \ldots, 0,1-v_{j 1}\right) \\
\begin{array}{l}
\text { with } u_{j}=\left(u_{j 1}, u_{j 2}\right), v_{j}=\left(v_{j 1}, v_{j 2}\right) \in \mathbb{R} \times \mathbb{R}^{n_{j}-1} \\
\text { satisfying } u_{j 1}, v_{j 1}<1 .
\end{array}
\end{gathered}
$$

The following proposition plays a key role in achieving the main result of this section.

Proposition 16. For $(\bar{x}, \bar{y})$ satisfying (82), let $\left[U_{j} V_{j}\right] \in$ $\partial \phi_{\mathrm{FB}}\left(\bar{x}_{j}, \bar{y}_{j}\right)$ for $j=1, \ldots, r$. Then, for any $(\Delta u)_{j},(\Delta v)_{j} \in \mathbb{R}^{n_{j}}$, it holds that

$U_{j}(\Delta u)_{j}+V_{j}(\Delta v)_{j}$

$$
=0 \Longrightarrow\left\{\begin{array}{cl}
(\Delta v)_{j}=0, & \text { if } j \in J_{I 0}, \\
(\Delta u)_{j}=0, & \text { if } j \in J_{0 I}, \\
\left\langle(\Delta u)_{j}, \bar{y}_{j}\right\rangle=0,\left\langle(\Delta v)_{j}, \bar{x}_{j}\right\rangle=0, & \text { if } j \in J_{B B}, \\
(\Delta u)_{j} \in \mathbb{R}\left(\bar{y}_{j 1},-\bar{y}_{j 2}\right), & \text { if } j \in J_{0 B}, \\
(\Delta v)_{j} \in \mathbb{R}\left(\bar{x}_{j 1},-\bar{x}_{j 2}\right), & \text { if } j \in J_{B 0} .
\end{array}\right.
$$


Particularly, for each $j \in J_{B B}$, the following implication also holds:

$$
\begin{aligned}
& U_{j}(\Delta u)_{j}+V_{j}(\Delta v)_{j} \\
& =0 \Longrightarrow\left\{\begin{array}{l}
\left\langle(\Delta u)_{j},(\Delta v)_{j}\right\rangle=\frac{\bar{y}_{j 1}}{\bar{x}_{j 1}}\left((\Delta u)_{j 1}^{2}-\left\|(\Delta u)_{j 2}\right\|^{2}\right) \\
\left\langle(\Delta u)_{j},(\Delta v)_{j}\right\rangle=\frac{\bar{x}_{j 1}}{\bar{y}_{j 1}}\left((\Delta v)_{j 1}^{2}-\left\|(\Delta v)_{j 2}\right\|^{2}\right)
\end{array}\right.
\end{aligned}
$$

Proof. Throughout the proof, let $\bar{w}_{j}=\bar{x}_{j}^{2}+\bar{y}_{j}^{2}$ and $\bar{z}_{j}=\sqrt{\bar{w}_{j}}$ for $j=1,2, \ldots, r$. We prove the conclusion by discussing five cases as shown in the following arguments.

Case $1\left(j \in J_{I 0}\right)$. In this case $\bar{z}_{j}=\bar{x}_{j} \in$ int $\mathscr{K}^{n_{j}}$. From Corollary 10(a), it follows that the function $\phi_{\mathrm{FB}}$ is continuously differentiable at $\left(\bar{x}_{j}, \bar{y}_{j}\right)$. Therefore,

$$
\begin{aligned}
\partial \phi_{\mathrm{FB}}\left(\bar{x}_{j}, \bar{y}_{j}\right) & =\left\{\mathscr{J} \phi_{\mathrm{FB}}\left(\bar{x}_{j}, \bar{y}_{j}\right)\right\} \\
& =\left\{\left[I-L_{\bar{z}_{j}}^{-1} L_{\bar{x}_{j}} I-L_{\bar{z}_{j}}^{-1} L_{\bar{y}_{j}}\right]\right\}=\left\{\left[\begin{array}{ll}
0 & I
\end{array}\right]\right\} .
\end{aligned}
$$

Then, $U_{j}=0$ and $V_{j}=I$. Together with $U_{j}(\Delta u)_{j}+V_{j}(\Delta v)_{j}=$ 0 , we get $(\Delta v)_{j}=0$.

Case $2\left(j \in J_{0 I}\right)$. Using the same arguments as in Case 1 readily yields $(\Delta u)_{j}=0$.

Case $3\left(j \in J_{B B}\right)$. Now $\bar{x}_{j} \in \mathbf{b d}^{+} \mathscr{K}^{n_{j}}$ and $\bar{y}_{j} \in \mathbf{b d}^{+} \mathscr{K}^{n_{j}}$. By (82) and Lemma 7, there exists $\rho_{j}>0$ such that $\bar{x}_{j 1}=\rho_{j} \bar{y}_{j 1}$ and $\bar{x}_{j 2}=-\rho_{j} \bar{y}_{j 2}$, and consequently,

$$
\begin{gathered}
\bar{w}_{j}=\left(\begin{array}{c}
2\left(1+\rho_{j}^{2}\right) \bar{y}_{j 1}^{2} \\
2\left(1-\rho_{j}^{2}\right) \bar{y}_{j 1} \bar{y}_{j 2}
\end{array}\right) \in \operatorname{int} \mathscr{K}^{n_{j}}, \\
\bar{z}_{j}=\left(\begin{array}{c}
\left(1+\rho_{j}\right) \bar{y}_{j 1} \\
\left(1-\rho_{j}\right) \bar{y}_{j 2}
\end{array}\right)=\bar{x}_{j}+\bar{y}_{j} .
\end{gathered}
$$

From Corollary 10(a), $\phi_{\mathrm{FB}}$ is continuously differentiable at $\left(\bar{x}_{j}, \bar{y}_{j}\right)$, and hence

$$
\begin{aligned}
& U_{j}=I-L_{\bar{z}_{j}}^{-1} L_{\bar{x}_{j}}=L_{\bar{z}_{j}}^{-1} L_{\bar{z}_{j}-\bar{x}_{j}}=L_{\bar{z}_{j}}^{-1} L_{\bar{y}_{j}}, \\
& V_{j}=I-L_{\bar{z}_{j}}^{-1} L_{\bar{y}_{j}}=L_{\bar{z}_{j}}^{-1} L_{\bar{z}_{j}-\bar{y}_{j}}=L_{\bar{z}_{j}}^{-1} L_{\bar{x}_{j}} .
\end{aligned}
$$

Thus, $U_{j}(\Delta u)_{j}+V_{j}(\Delta v)_{j}=0$ implies that $L_{\bar{y}_{j}}(\Delta u)_{j}+$ $L_{\bar{x}_{j}}(\Delta v)_{j}=0$, and consequently,

$$
\begin{aligned}
& \left(\bar{x}_{j 1}(\Delta v)_{j 1}+\bar{x}_{j 2}^{T}(\Delta v)_{j 2}\right)+\left(\bar{y}_{j 1}(\Delta u)_{j 1}+\bar{y}_{j 2}^{T}(\Delta u)_{j 2}\right)=0 \\
& \left(\bar{x}_{j 2}(\Delta v)_{j 1}+\bar{x}_{j 1}(\Delta v)_{j 2}\right)+\left(\bar{y}_{j 2}(\Delta u)_{j 1}+\bar{y}_{j 1}(\Delta u)_{j 2}\right)=0 .
\end{aligned}
$$

Making an inner product with $\bar{y}_{j 2} /\left\|\bar{y}_{j 2}\right\|$, we have from $\bar{x}_{j}, \bar{y}_{j} \in \mathbf{b d}^{+} \mathscr{K}^{n_{j}}$ and Lemma 5 that

$$
-\left(\bar{x}_{j 1}(\Delta v)_{j 1}+\bar{x}_{j 2}^{T}(\Delta v)_{j 2}\right)+\left(\bar{y}_{j 1}(\Delta u)_{j 1}+\bar{y}_{j 2}^{T}(\Delta u)_{j 2}\right)=0 .
$$

Together with (94), we have $\left\langle(\Delta u)_{j}, \bar{y}_{j}\right\rangle=0$ and $\left\langle(\Delta v)_{j}, \bar{x}_{j}\right\rangle=$ 0 .

We next prove the implication in (90). By the expressions of $\bar{z}_{j}, U_{j}$, and $V_{j}$,

$$
\begin{aligned}
& U_{j}(\Delta u)_{j}+V_{j}(\Delta v)_{j}=0 \\
& \Longleftrightarrow\left[\begin{array}{cc}
\bar{y}_{j 1} & \bar{y}_{j 2}^{T} \\
\bar{y}_{j 2} & \bar{y}_{j 1} I
\end{array}\right](\Delta u)_{j}+\left[\begin{array}{cc}
\bar{x}_{j 1} & \bar{x}_{j 2}^{T} \\
\bar{x}_{j 2} & \bar{x}_{j 1} I
\end{array}\right](\Delta v)_{j}=0 \\
& \Longleftrightarrow\left[\begin{array}{ll}
\bar{y}_{j 1} & \bar{y}_{j 2}^{T} \\
\bar{y}_{j 2} & \bar{y}_{j 1} I
\end{array}\right](\Delta u)_{j}+\frac{\bar{x}_{j 1}}{\bar{y}_{j 1}}\left[\begin{array}{cc}
\bar{y}_{j 1} & -\bar{y}_{j 2}^{T} \\
-\bar{y}_{j 2} & \bar{y}_{j 1} I
\end{array}\right](\Delta v)_{j}=0 \\
& \Longrightarrow\left[\bar{y}_{j 2} \quad \bar{y}_{j 1} I\right](\Delta u)_{j}+\frac{\bar{x}_{j 1}}{\bar{y}_{j 1}}\left[-\bar{y}_{j 2} \bar{y}_{j 1} I\right](\Delta v)_{j}=0 \\
& \Longleftrightarrow\left[\begin{array}{ll}
-\bar{y}_{j 2} & \bar{y}_{j 1} I
\end{array}\right]\left[\begin{array}{l}
\bar{x}_{j 1} \\
\bar{y}_{j 1}
\end{array}\left(\begin{array}{c}
(\Delta v)_{j 1} \\
(\Delta v)_{j 2}
\end{array}\right)+\left(\begin{array}{c}
-(\Delta u)_{j 1} \\
(\Delta u)_{j 2}
\end{array}\right)\right]=0,
\end{aligned}
$$

where the second equivalence is due to $\bar{x}_{j 1}=\rho_{j} \bar{y}_{j 1}$ and $\bar{x}_{j 2}=$ $-\rho_{j} \bar{y}_{j 2}$. Since the rank of $\left[-\bar{y}_{j 2} \quad \bar{y}_{j 1} I\right]$ is $n_{j}-1$, the dimension of the solution space for the system $\left[-\bar{y}_{j 2} \quad \bar{y}_{j 1} I\right] \zeta=0$ equals 1. Note that $\bar{y}_{j}$ is a nonzero solution of this linear system. Therefore,

$$
\frac{\bar{x}_{j 1}}{\bar{y}_{j 1}}\left(\begin{array}{c}
(\Delta v)_{j 1} \\
(\Delta v)_{j 2}
\end{array}\right)+\left(\begin{array}{c}
-(\Delta u)_{j 1} \\
(\Delta u)_{j 2}
\end{array}\right)=c \bar{y}_{j} \quad \text { for some } c \in \mathbb{R} .
$$

Making an inner product with $(\Delta u)_{j}$ for the equality and using $\left\langle(\Delta u)_{j}, \bar{y}_{j}\right\rangle=0$, we get

$$
\frac{\bar{x}_{j 1}}{\bar{y}_{j 1}}\left\langle(\Delta u)_{j},(\Delta v)_{j}\right\rangle=(\Delta u)_{j 1}^{2}-\left\|(\Delta u)_{j 2}\right\|^{2} .
$$

Using the similar arguments as above and noting that $\left\langle(\Delta v)_{j}, \bar{x}_{j}\right\rangle=0$, we may obtain

$$
\frac{\bar{y}_{j 1}}{\bar{x}_{j 1}}\left\langle(\Delta u)_{j},(\Delta v)_{j}\right\rangle=(\Delta v)_{j 1}^{2}-\left\|(\Delta v)_{j 2}\right\|^{2} .
$$

The last two equalities show that the implication in (90) holds.

Case $4\left(j \in J_{0 B}\right)$. By Lemma 15(b), there exists an $n_{j} \times n_{j}$ orthogonal matrix $Q_{j}=\left[\begin{array}{lll}q_{j} & \widehat{Q}_{j} & q_{j}^{\prime}\end{array}\right]$ such that $U_{j}=Q_{j} D_{j} Q_{j}^{T}$ and $V_{j}=Q_{j} \Lambda_{j} Q_{j}^{T}$, where $q_{j}$ and $q_{j}^{\prime}$ are given by (86), and $Q_{j}$ and $\Lambda_{j}$ take one of the form in (87)-(88). Thus, we have

$$
U_{j}(\Delta u)_{j}+V_{j}(\Delta v)_{j}=0 \Longleftrightarrow D_{j} Q_{j}^{T}(\Delta u)_{j}+\Lambda_{j} Q_{j}^{T}(\Delta v)_{j}=0 .
$$


When $D_{j}=I$ and $\Lambda_{j}=0$, we have $Q_{j}^{T}(\Delta u)_{j}=0$, and then $(\Delta u)_{j}=0$. When $D_{j}$ and $\Lambda_{j}$ take the form in (87), we have $\left[\begin{array}{c}q_{j}^{T} \\ \widehat{Q}_{j}^{T}\end{array}\right](\Delta u)_{j}=0$. Consequently,

$$
\begin{aligned}
(\Delta u)_{j} & =Q_{j} Q_{j}^{T}(\Delta u)_{j}=\left[\begin{array}{lll}
q_{j} & \widehat{Q}_{j} & q_{j}^{\prime}
\end{array}\right]\left[\begin{array}{c}
0 \\
0 \\
\left(q_{j}^{\prime}\right)^{T}(\Delta u)_{j}
\end{array}\right] \\
& =\frac{\left(q_{j}^{\prime}\right)^{T}(\Delta u)_{j}}{\sqrt{2} \bar{y}_{j 1}}\left[\begin{array}{c}
\bar{y}_{j 1} \\
-\bar{y}_{j 2}
\end{array}\right],
\end{aligned}
$$

where the last equality is using the definition of $q_{j}^{\prime}$. When $D_{j}$ and $\Lambda_{j}$ take the form in (88), we also have $\left[\begin{array}{c}q_{j}^{T} \\ \widetilde{Q}_{j}^{T}\end{array}\right](\Delta u)_{j}=0$. Using the same arguments as above, we have that $(\Delta u)_{j}$ has the form of (102). Thus, we prove that $(\Delta u)_{j} \in \mathbb{R}\left(\bar{y}_{j 1}, \bar{y}_{j 2}\right)$.

Case $5\left(j \in J_{B 0}\right)$. Using Lemma 15(a) and following the same arguments as in Case 4, the result can be checked routinely. So, we omit the proof.

The following lemma states an important property for the elements of Clarke's Jacobian of $\phi_{\mathrm{FB}}$ at a general point, which will be used to prove Proposition 18.

Lemma 17. For any given $(x, y) \in \mathbb{R}^{n} \times \mathbb{R}^{n}$, let $[U V] \in$ $\partial \phi_{\mathrm{FB}}(x, y)$. Then,

$$
U \Delta u+V \Delta v=0 \Longrightarrow\langle\Delta u, \Delta v\rangle \leq 0, \quad \forall \Delta u, \Delta v \in \mathbb{R}^{n}
$$

Proof. Since $[U V] \in \partial \phi_{\mathrm{FB}}(x, y)$, by Carathéodory's theo-

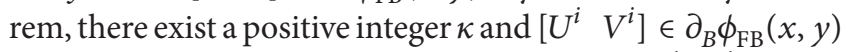
for $i=1, \ldots, \kappa$ such that $[U V]=\sum_{i=1}^{\kappa} \tau_{i}\left[U^{i} V^{i}\right]$ where $\tau_{i} \geq 0, i=1, \ldots, \kappa$, and $\sum_{i=1}^{\kappa} \tau_{i}=1$. For each $i \in\{1, \ldots, \kappa\}$, by Corollary 10(a) and the definition of the elements in $\partial_{B} \phi_{\mathrm{FB}}(x, y)$, there exists a sequence $\left\{\left(x^{i_{k}}, y^{i_{k}}\right)\right\}$ in $\mathbb{R}^{n} \times \mathbb{R}^{n}$ converging to $(x, y)$ with $z^{i_{k}}=z\left(x^{i_{k}}, y^{i_{k}}\right)>0$ such that

$$
\begin{aligned}
{\left[\begin{array}{ll}
U^{i} & V^{i}
\end{array}\right] } & =\lim _{k \rightarrow \infty} \mathcal{J} \phi_{\mathrm{FB}}\left(x^{i_{k}}, y^{i_{k}}\right) \\
& =\lim _{k \rightarrow \infty}\left[I-L_{z^{i_{k}}}^{-1} L_{x^{i_{k}}} I-L_{z^{i_{k}}}^{-1} L_{y^{i_{k}}}\right] .
\end{aligned}
$$

Consequently, for any $\Delta u, \Delta v \in \mathbb{R}^{n}$, we have that

$$
\begin{aligned}
&(I-U) \Delta u+(I-V) \Delta v \\
& \quad=\lim _{k \rightarrow \infty} \sum_{i=1}^{\kappa} \tau_{i}\left(L_{z^{k} k}^{-1} L_{x^{i} k} \Delta u+L_{z^{k} k}^{-1} L_{y^{i k}} \Delta v\right) .
\end{aligned}
$$

From the continuity and convexity of the Euclidean norm $\|\cdot\|$ and Lemma 4, we get

$$
\begin{aligned}
& \|(I-U) \Delta u+(I-V) \Delta v\| \\
& \quad \leq \lim _{k \rightarrow \infty} \sum_{i=1}^{\kappa} \tau_{i}\left\|\left(L_{z^{i_{k}}}^{-1} L_{x^{i_{k}}} \Delta u+L_{z^{i_{k}}}^{-1} L_{y^{i_{k}}} \Delta v\right)\right\| \\
& \leq \sqrt{\|\Delta u\|^{2}+\|\Delta v\|^{2}} .
\end{aligned}
$$

Now assume that $U \Delta u+V \Delta v=0$. Then, by the last inequality, we get the result.

Proposition 18. For $(\bar{x}, \bar{y})$ satisfying (82), let $\left[\begin{array}{ll}U & V\end{array}\right] \in$ $\partial \phi_{\mathrm{FB}}(\bar{x}, \bar{y})$. Then it holds that

$$
\begin{aligned}
U \Delta u+V \Delta v & =0 \Longrightarrow\langle\Delta u, \Delta v\rangle \\
& \leq \sum_{j=1}^{r} \Upsilon_{\bar{x}_{j}}\left(\bar{y}_{j},(\Delta u)_{j}\right) \quad \forall \Delta u, \Delta v \in \mathbb{R}^{n},
\end{aligned}
$$

where, for any given $\Phi \in \mathbb{R}^{n}, \Upsilon_{\varrho_{j}}: \mathbb{R}^{n_{j}} \times \mathbb{R}^{n_{j}} \rightarrow \mathbb{R}$ is the linear-quadratic function:

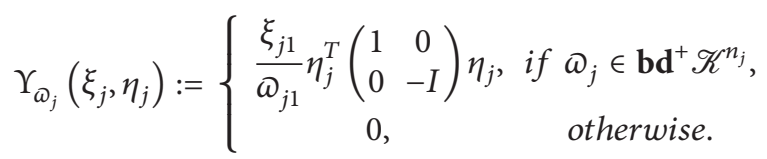

Proof. Fix any $\Delta u, \Delta v \in \mathbb{R}^{n}$. Since $U=\operatorname{diag}\left(U_{1}, \ldots, U_{r}\right)$ and $V=\operatorname{diag}\left(V_{1}, \ldots, V_{r}\right)$ with $\left[U_{j} V_{j}\right] \in \partial \phi_{\mathrm{FB}}\left(\bar{x}_{j}, \bar{y}_{j}\right)$ for $j=$ $1,2, \ldots, r$, we may rewrite $U \Delta u+V \Delta v=0$ as

$$
U_{j}(\Delta u)_{j}+V_{j}(\Delta v)_{j}=0, \quad j=1,2, \ldots, r .
$$

From Lemma 17 and (90) of Proposition 16, it then follows that

$$
\begin{aligned}
\langle\Delta u, \Delta v\rangle & \leq \sum_{j \in J_{B B}}\left\langle(\Delta u)_{j},(\Delta v)_{j}\right\rangle \\
& =\sum_{j \in J_{B B}} \frac{\bar{y}_{j 1}}{\bar{x}_{j 1}}\left[(\Delta u)_{j 1}^{2}-\left\|(\Delta u)_{j 2}\right\|^{2}\right] .
\end{aligned}
$$

In addition, by the definition of $\Upsilon_{\omega_{j}}, \Upsilon_{\bar{x}_{j}}\left(\bar{y}_{j}, \cdot\right)=0$ for all $j \epsilon$ $J_{I 0} \cup J_{0 I} \cup J_{0 B} \cup J_{00}$ since $\bar{x}_{j} \notin \mathbf{b d}^{+} \mathscr{K}^{n_{j}}$, and $\Upsilon_{\bar{x}_{j}}\left(\bar{y}_{j}, \cdot\right)=0$ for $j \in J_{B 0}$ since $\bar{y}_{j 1}=0$. This means that

$$
\begin{aligned}
\sum_{j=1}^{r} \Upsilon_{\bar{x}_{j}}\left(\bar{y}_{j},(\Delta u)_{j}\right) & =\sum_{j \in J_{B B}} \Upsilon_{\bar{x}_{j}}\left(\bar{y}_{j},(\Delta u)_{j}\right) \\
& =\sum_{j \in J_{B B}} \frac{\bar{y}_{j 1}}{\bar{x}_{j 1}}\left[(\Delta u)_{j 1}^{2}-\left\|(\Delta u)_{j 2}\right\|^{2}\right] .
\end{aligned}
$$

From the above two equations, we immediately obtain the desired result. 
Before stating the main result of this section, we also need to recall several concepts, including constraint nondegeneracy, Robinson's constraint qualification (CQ) (see [20]), and the strong second-order sufficient condition introduced in [7, Theorem 30]. To this end, let $\omega \equiv(\zeta, x) \in \mathbb{R}^{2 n}$ and define $\tilde{f}: \mathbb{R}^{2 n} \rightarrow \mathbb{R}, \tilde{h}: \mathbb{R}^{2 n} \rightarrow \mathbb{R}^{n+m}, \tilde{g}: \mathbb{R}^{2 n} \rightarrow \mathbb{R}^{n}$ by

$$
\tilde{f}(\omega):=f(\zeta), \quad \tilde{h}(\omega):=\left(\begin{array}{c}
h(\zeta) \\
g(\zeta)-x
\end{array}\right), \quad \tilde{g}(\omega):=x .
$$

Then, we may rewrite the nonlinear SOCP (4) succinctly as follows:

$$
\begin{array}{cl}
\min _{\omega \in \mathbb{R}^{2 n}} & \tilde{f}(\omega) \\
\text { s.t. } & \tilde{h}(\omega)=0, \\
& \widetilde{g}(\omega) \in \mathscr{K} .
\end{array}
$$

Definition 19. A feasible vector $\bar{\omega}=(\bar{\zeta}, \bar{x})$ of (4) is called constraint nondegenerate if

$$
\left(\begin{array}{c}
\mathscr{J}_{\omega} \widetilde{h}(\bar{\omega}) \\
\mathscr{J}_{\omega} \widetilde{g}(\bar{\omega})
\end{array}\right) \mathbb{R}^{2 n}+\left(\begin{array}{c}
\{0\} \\
\operatorname{lin}\left(\mathscr{T}_{\mathscr{K}}(\bar{x})\right)
\end{array}\right)=\left(\begin{array}{c}
\mathbb{R}^{m+n} \\
\mathbb{R}^{n}
\end{array}\right),
$$

where $\operatorname{lin}\left(\mathscr{T}_{\mathscr{K}}(\bar{x})\right)$ is the largest linear space of $\mathscr{T}_{\mathscr{K}}(\bar{x})$, that is, $\operatorname{lin}\left(\mathscr{T}_{\mathscr{K}}(\bar{x})\right)=\mathscr{T}_{\mathscr{K}}(\bar{x}) \cap-\mathscr{T}_{\mathscr{K}}(\bar{x})$.

Definition 20. Robinson's CQ is said to hold at a feasible solution $\bar{\omega}=(\bar{\zeta}, \bar{x})$ to $(4)$ if

$$
0 \in \operatorname{int}\left\{\left(\begin{array}{l}
\tilde{h}(\bar{\omega}) \\
\widetilde{g}(\bar{\omega})
\end{array}\right)+\left(\begin{array}{l}
\mathscr{J}_{\omega} \tilde{h}(\bar{\omega}) \\
\mathscr{J}_{\omega} \widetilde{g}(\bar{\omega})
\end{array}\right) \mathbb{R}^{2 n}-\left(\begin{array}{c}
\{0\} \\
\mathscr{K}
\end{array}\right)\right\},
$$

which, since $\mathscr{K}$ is a closed convex set in $\mathbb{R}^{n}$, can be equivalently written as

$$
\left(\begin{array}{c}
\mathscr{J}_{\omega} \tilde{h}(\bar{\omega}) \\
\mathscr{J}_{\omega} \widetilde{g}(\bar{\omega})
\end{array}\right) \mathbb{R}^{2 n}+\left(\begin{array}{c}
\{0\} \\
\mathscr{T}_{\mathscr{K}}(\bar{x})
\end{array}\right)=\left(\begin{array}{c}
\mathbb{R}^{m+n} \\
\mathbb{R}^{n}
\end{array}\right) .
$$

Clearly, the constraint nondegenerate condition (114) implies Robinson's CQ (116). If $\bar{\omega}=(\bar{\zeta}, \bar{x})$ is a locally optimal solution to (4) and Robinson's CQ holds at $\bar{\omega}$, then there exists a Lagrange multiplier $(\bar{\mu}, \bar{s}, \bar{y})$, together with $\bar{\omega}$, satisfying the KKT conditions:

$$
\begin{gathered}
\mathscr{J}_{\zeta, x} L(\bar{\zeta}, \bar{x}, \bar{\mu}, \bar{s}, \bar{y})=0, \quad h(\bar{\zeta})=0, \\
g(\bar{\zeta})-\bar{x}=0, \quad-\bar{y} \in \mathcal{N}_{\mathscr{K}}(\bar{x}) .
\end{gathered}
$$

In the sequel, we let $\Lambda(\bar{\omega})$ denote the set of Lagrange multipliers satisfying (117).

Let $(\bar{\zeta}, \bar{x}, \bar{\mu}, \bar{s}, \bar{y}) \in \mathbb{R}^{n} \times \mathscr{K} \times \mathbb{R}^{m} \times \mathbb{R}^{n} \times \mathscr{K}$ be a KKT point of the SOCP (4). From [7, Lemma 25], it follows that the tangent cone of $\mathscr{K}$ at $\bar{x}$ takes the form of

$$
\begin{gathered}
\mathscr{T}_{\mathscr{K}}(\bar{x})=\left\{d \in \mathbb{R}^{n} \mid d_{j} \in \mathscr{K}^{n_{j}} \text { for } j \in J_{0 I} \cup J_{0 B} \cup J_{00} ;\right. \\
\left.d_{j}^{T}\left(\bar{x}_{j 1},-\bar{x}_{j 2}\right) \geq 0 \text { for } j \in J_{B B} \cup J_{B 0}\right\},
\end{gathered}
$$

which implies that the largest linear space in $\mathscr{T}_{\mathscr{K}}(\bar{x})$ has the following form:

$$
\begin{aligned}
& \operatorname{lin}\left(\mathscr{T}_{\mathscr{K}}(\bar{x})\right) \\
& =\left\{d \in \mathbb{R}^{n} \mid d_{j}=0 \text { for } j \in J_{0 I} \cup J_{0 B} \cup J_{00} ;\right. \\
& \left.\quad d_{j}^{T}\left(\bar{x}_{j 1},-\bar{x}_{j 2}\right)=0 \text { for } j \in J_{B B} \cup J_{B 0}\right\} .
\end{aligned}
$$

We next recall the critical cone of problem (4) at a feasible $\omega^{0}$ which is defined as

$$
\begin{gathered}
\mathscr{C}\left(\omega^{0}\right):=\left\{d \in \mathbb{R}^{2 n} \mid \mathscr{J}_{\omega} \tilde{h}\left(\omega^{0}\right) d=0,\right. \\
\mathscr{J}_{\omega} \widetilde{\mathscr{g}}\left(\omega^{0}\right) d \in \mathscr{T}_{\mathscr{K}}\left(\widetilde{g}\left(\omega^{0}\right)\right), \\
\left.\mathscr{J}_{\omega} \tilde{f}\left(\omega^{0}\right) d \leq 0\right\} .
\end{gathered}
$$

The critical cone $\mathscr{C}\left(\omega^{0}\right)$ represents those directions for which the linearization of (4) does not provide any information about optimality of $\omega^{0}$ and is very important in studying second-order optimality conditions. Particularly, if the set of Lagrange multipliers $\Lambda\left(\omega^{0}\right)$ at the point $\omega^{0}$ is nonempty, then $\mathscr{C}\left(\omega^{0}\right)$ can be rewritten as

$$
\begin{aligned}
\mathscr{C}\left(\omega^{0}\right) & \\
= & \left\{d \in \mathbb{R}^{2 n} \mid \mathscr{J}_{\omega} \tilde{h}\left(\omega^{0}\right) d=0,\right. \\
& \left.\mathscr{J}_{\omega} \widetilde{g}\left(\omega^{0}\right) d \in \mathscr{T}_{\mathscr{K}}\left(\widetilde{g}\left(\omega^{0}\right)\right) \cap\left(y^{0}\right)^{\perp}\right\},
\end{aligned}
$$

where $y^{0} \in \Lambda\left(\omega^{0}\right)$ and $\left(y^{0}\right)^{\perp}$ means the orthogonal complementarity space of $y^{0}$. Now let $(\bar{\mu}, \bar{s}, \bar{y}) \in \Lambda(\bar{\omega})$. Then, using $\tilde{g}(\bar{\omega})=\bar{x}$ and the expression of $\mathscr{T}_{\mathscr{K}}(\bar{x})$, we have that

$$
\begin{gathered}
\mathscr{C}(\bar{\omega})=\left\{d \in \mathbb{R}^{2 n} \mid \mathscr{J}_{\omega} \tilde{g}_{j}(\bar{\omega}) d=0 \text { for } j \in J_{0 I},\right. \\
\mathscr{J}_{\omega} \widetilde{g}_{j}(\bar{\omega}) d \in \mathbb{R}_{+}\left(\bar{y}_{j 1},-\bar{y}_{j 2}\right) \\
\quad \text { for } j \in J_{0 B}, \\
\left\langle\mathscr{J}_{\omega} \tilde{g}_{j}(\bar{\omega}) d, \bar{y}_{j}\right\rangle=0 \text { for } j \in J_{B B} \\
\mathscr{J}_{\omega} \widetilde{g}_{j}(\bar{\omega}) d \in \mathscr{K}^{n_{j}} \text { for } j \in J_{00} \\
\left(\mathscr{J}_{\omega} \tilde{g}_{j}(\bar{\omega}) d\right)\left(\bar{x}_{j 1},-\bar{x}_{j 2}\right) \geq 0 \\
\quad \text { for } j \in J_{B 0} \\
\left.\mathscr{J}_{\omega} \tilde{h}(\bar{\omega}) d=0\right\} .
\end{gathered}
$$

Definition 21. Let $\bar{\omega}=(\bar{\zeta}, \bar{x})$ be a stationary point of the SOCP (4) such that $\Lambda(\bar{\omega})=\{(\bar{\mu}, \bar{s}, \bar{y})\}$. We say that the strong second-order sufficient condition holds at $\bar{\omega}$ if

$$
\begin{aligned}
&\left\langle d, \mathscr{J}_{\omega \omega}^{2} L(\bar{\zeta}, \bar{x}, \bar{\mu}, \bar{s}, \bar{y}) d\right\rangle+\langle d, H(\bar{\omega}, \bar{y}) d\rangle>0, \\
& \forall d \in \operatorname{aff}(\mathscr{C}(\bar{\omega})) \backslash\{0\},
\end{aligned}
$$


where $H(\bar{\omega}, \bar{y})=\sum_{j=1}^{r} H_{j}(\bar{\omega}, \bar{y}) \in \mathbb{R}^{2 n \times 2 n}$ with $H_{j}(\bar{\omega}, \bar{y}) \in$ $\mathbb{R}^{2 n \times 2 n}$ defined by

$$
\begin{aligned}
& H_{j}(\bar{\omega}, \bar{y}) \\
& :=\left\{\begin{array}{cc}
-\bar{y}_{j 1} \mathscr{J}_{\omega} \widetilde{g}_{j}(\bar{\omega})^{T}\left(\begin{array}{cc}
1 & 0 \\
0 & -I
\end{array}\right) \mathscr{J}_{\omega} \tilde{g}_{j}(\bar{\omega}), & \text { if } j \in J_{B B} \cup J_{B 0} ; \\
0, & \text { otherwise, }
\end{array}\right.
\end{aligned}
$$

and aff $(\mathscr{C}(\bar{\omega}))$ denotes the affine hull of $\mathscr{C}(\bar{\omega})$ and is now equivalent to the span of $\mathscr{C}(\bar{\omega})$ :

$$
\begin{aligned}
& \operatorname{aff}(\mathscr{C}(\bar{\omega})) \\
& =\left\{d \in \mathbb{R}^{2 n} \mid \mathscr{J}_{\omega} \widetilde{h}(\bar{\omega}) d=0 ; \mathscr{F}_{\omega} \widetilde{\mathcal{g}}_{j}(\bar{\omega}) d=0 \text { for } j \in J_{0 I},\right. \\
& \left\langle\mathscr{J}_{\omega} \widetilde{g}_{j}(\bar{\omega}) d, \bar{y}_{j}\right\rangle=0 \text { for } j \in J_{B B}, \\
& \left.\mathscr{J}_{\omega} \tilde{g}_{j}(\bar{\omega}) d \in \mathbb{R}\left(\bar{y}_{j 1},-\bar{y}_{j 2}\right) \text { for } j \in J_{0 B}\right\} .
\end{aligned}
$$

Now we are in a position to prove the nonsingularity of Clarke's Jacobian of $E_{\mathrm{FB}}$ under the strong second-order sufficient condition and constraint nondegeneracy.

Proposition 22. Let $(\bar{\zeta}, \bar{x}, \bar{\mu}, \bar{s}, \bar{y})$ be a KKT point of the nonlinear SOCP (4). Suppose that the strong second-order sufficient condition (123) holds at $\bar{\omega}=(\bar{\zeta}, \bar{x})$ and $\bar{\omega}$ is constraint nondegenerate, then any element in $\partial E_{\mathrm{FB}}(\bar{\zeta}, \bar{x}, \bar{\mu}, \bar{s}, \bar{y})$ is nonsingular.

Proof. Since the nondegeneracy condition (114) is assumed to hold at $(\bar{\zeta}, \bar{x})$, from [21, Proposition 4.75], we know that $\Lambda(\bar{\omega})=\{(\bar{\mu}, \bar{s}, \bar{y})\}$. Then, by the definition of $L$ and $\tilde{g}$, the strong second-order sufficient condition (123) takes the following form:

$$
\begin{array}{r}
\left\langle\xi, \mathscr{J}_{\zeta \zeta}^{2} l(\bar{\zeta}, \bar{\mu}, \bar{s}) \xi\right\rangle-\langle\eta, \widehat{H}(\bar{x}, \bar{y}) \eta\rangle>0, \\
\forall(\xi, \eta) \in \operatorname{aff}(\mathscr{C}(\bar{\omega})) \backslash\{(0,0)\},
\end{array}
$$

where $l(\zeta, \mu, s):=f(\zeta)+\langle\mu, h(\zeta)\rangle+\langle g(\zeta), s\rangle$ and $\widehat{H}(\bar{x}, \bar{y})=$ $\sum_{j=1}^{r} \widehat{H}_{j}(\bar{x}, \bar{y}) \in \mathbb{R}^{n \times n}$ with

$$
\widehat{H}_{j}(\bar{x}, \bar{y}):=\left\{\begin{array}{cc}
-\bar{y}_{j 1}\left(\begin{array}{cc}
1 & 0 \\
\bar{x}_{j 1} & -I
\end{array}\right), & \text { if } j \in J_{B B} \cup J_{B 0} ; \\
0, & \text { otherwise. }
\end{array}\right.
$$

Let $W$ be an arbitrary element in $\partial E_{\mathrm{FB}}(\bar{\zeta}, \bar{x}, \bar{\mu}, \bar{s}, \bar{y})$. To prove that $W$ is nonsingular, let $(\Delta \zeta, \Delta x, \Delta \mu, \Delta s, \Delta y) \in \mathbb{R}^{n} \times \mathbb{R}^{n} \times$ $\mathbb{R}^{m} \times \mathbb{R}^{n} \times \mathbb{R}^{n}$ such that

$$
W(\Delta \zeta, \Delta x, \Delta \mu, \Delta s, \Delta y)=0 .
$$

From the expression of $E_{\mathrm{FB}}$, we know that there exists a $\left[\begin{array}{ll}U & V\end{array}\right] \in \partial \phi_{\mathrm{FB}}(\bar{x}, \bar{y})$ such that

$$
\left[\begin{array}{c}
\mathscr{F}_{\zeta \zeta} l(\bar{\zeta}, \bar{\mu}, \bar{s}) \Delta \zeta+\mathscr{F}_{\zeta} h(\bar{\zeta})^{T} \Delta \mu+\mathscr{F}_{\zeta} g(\bar{\zeta})^{T} \Delta s \\
-\Delta s-\Delta y \\
\mathcal{F}_{\zeta} h(\bar{\zeta}) \Delta \zeta \\
\mathscr{F}_{\zeta} g(\bar{\zeta}) \Delta \zeta-\Delta x \\
U \Delta x+V \Delta y
\end{array}\right]=0
$$

where $U=\operatorname{diag}\left(U_{1}, \ldots, U_{r}\right)$ and $V=\operatorname{diag}\left(V_{1}, \ldots, V_{r}\right)$ with $\left[U_{j} V_{j}\right] \in \partial \phi_{\mathrm{FB}}\left(\bar{x}_{j}, \bar{y}_{j}\right)$ for all $j=1,2, \ldots, r$. The last system can be simplified as

$$
\left[\begin{array}{c}
\mathscr{F}_{\zeta \zeta}^{2} l(\bar{\zeta}, \bar{\mu}, \bar{s}) \Delta \zeta+\mathscr{F}_{\zeta} h(\bar{\zeta})^{T} \Delta \mu-\mathcal{F}_{\zeta} g(\bar{\zeta})^{T} \Delta y \\
\mathscr{F}_{\zeta} h(\bar{\zeta}) \Delta \zeta \\
U \mathscr{F}_{\zeta} g(\bar{\zeta}) \Delta \zeta+V \Delta y
\end{array}\right]=0 .
$$

By the second and the third equations of (130) and (89), we get

$$
\begin{gathered}
\mathscr{J}_{\zeta} h(\bar{\zeta}) \Delta \zeta=0, \\
\left(\mathscr{J}_{\zeta} g(\bar{\zeta}) \Delta \zeta\right)_{j}=\mathbb{R}\left(\bar{y}_{j 1},-\bar{y}_{j 2}\right), \quad \text { for } j \in J_{0 B}, \\
\left(\mathscr{J}_{\zeta} g(\bar{\zeta}) \Delta \zeta\right)_{j}=0, \quad \text { for } j \in J_{0 I}, \\
\left\langle\left(\mathscr{F}_{\zeta} g(\bar{\zeta}) \Delta \zeta\right)_{j}, \bar{y}_{j}\right\rangle=0, \quad \text { for } j \in J_{B B} .
\end{gathered}
$$

Comparing with the definition of aff $(\mathscr{C}(\bar{\omega}))$ in Definition 21, it follows that

$$
\left(\Delta \zeta, \mathscr{F}_{\zeta} g(\bar{\zeta}) \Delta \zeta\right) \in \operatorname{aff}(\mathscr{C}(\bar{\omega}))
$$

From the first and the second equations of (130), it is not hard to verify

$$
\begin{aligned}
0 & =\left\langle\Delta \zeta, \mathscr{F}_{\zeta \zeta}^{2} l(\bar{\zeta}, \bar{\mu}, \bar{s}) \Delta \zeta+\mathscr{F}_{\zeta} h(\bar{\zeta})^{T} \Delta \mu-\mathscr{F}_{\zeta} g(\bar{\zeta})^{T} \Delta y\right\rangle \\
& =\left\langle\Delta \zeta, \mathscr{F}_{\zeta \zeta}^{2} l(\bar{\zeta}, \bar{\mu}, \bar{s}) \Delta \zeta\right\rangle-\left\langle\mathscr{F}_{\zeta} g(\bar{\zeta}) \Delta \zeta, \Delta y\right\rangle,
\end{aligned}
$$

which, together with the third equation of (130) and Proposition 18, implies that

$$
0 \geq\left\langle\Delta \zeta, \mathscr{J}_{\zeta \zeta}^{2} l(\bar{\zeta}, \bar{\mu}, \bar{s}) \Delta \zeta\right\rangle-\sum_{j=1}^{r} \Upsilon_{\bar{x}_{j}}\left(\bar{y}_{j}, \mathscr{J}_{\zeta} g_{j}(\bar{\zeta}) \Delta \zeta\right)
$$

This, together with (132) and (126), yields that $\Delta \zeta=0$. Thus, (130) reduces to

$$
\left[\begin{array}{c}
\mathscr{F}_{\zeta} h(\bar{\zeta})^{T} \Delta \mu-\mathscr{F}_{\zeta} g(\bar{\zeta})^{T} \Delta y \\
V \Delta y
\end{array}\right]=0 .
$$

From the second equation of (135), we have $V_{j}(\Delta y)_{j}=0$ for $j \in J_{B B}$. In addition, by the arguments for Case 3 of 
Proposition 16, $V_{j}=L_{\bar{z}_{j}}^{-1} L_{\bar{x}_{j}}$ for $j \in J_{B B}$, and so $L_{\bar{x}_{j}}(\Delta y)_{j}=0$ for $j \in J_{B B}$. Since $\bar{x}_{j} \in \mathbf{b d}^{+} \mathscr{K}^{n_{j}}$ for $j \in J_{B B}, L_{\bar{x}_{j}}$ has the two single eigenvalues 0 and $2 \bar{x}_{j 1}$ as well as the eigenvalues $\bar{x}_{j 1}$ with multiplicity $n_{j}-2$, and $\left(1,-\bar{x}_{j 2} / \bar{x}_{j 1}\right)$ is the eigenvector corresponding to eigenvalue 0 . Thus, from $L_{\bar{x}_{j}}(\Delta y)_{j}=0$, we deduce

$$
(\Delta y)_{j} \in \mathbb{R}\left(x_{j 1},-\bar{x}_{j 2}\right), \quad \text { for } j \in J_{B B} .
$$

By the second equation of (135), we use Proposition 16 with $\Delta u=0, \Delta v=\Delta y$ to yield

$$
\begin{gathered}
(\Delta y)_{j}=0, \quad \text { for } j \in J_{I 0}, \\
(\Delta y)_{j} \in \mathbb{R}\left(\bar{x}_{j 1},-\bar{x}_{j 2}\right), \quad \text { for } j \in J_{B 0} .
\end{gathered}
$$

Using the constraint nondegeneracy condition (114), we know that there exist a vector $\left(d_{1}, d_{2}\right) \in \mathbb{R}^{n} \times \mathbb{R}^{n}$ and a vector $v \in$ $\operatorname{lin}\left(\mathscr{T}_{\mathscr{K}}(\bar{x})\right)$ such that

$$
\begin{gathered}
\mathscr{J}_{\zeta} h(\bar{\zeta}) d_{1}=\Delta \mu, \quad \mathscr{J}_{\zeta} g(\bar{\zeta}) d_{1}-d_{2}=-\Delta y, \\
d_{2}+v=-\Delta y .
\end{gathered}
$$

Since $v \in \operatorname{lin}\left(\mathscr{T}_{\mathscr{K}}(\bar{x})\right)$, from (119), it follows that

$$
\begin{gathered}
v_{j}=0, \quad \text { for } j \in J_{0 I} \cup J_{0 B} \cup J_{00}, \\
v_{j}^{T}\left(\bar{x}_{j 1},-\bar{x}_{j 2}\right)=0, \quad \text { for } j \in J_{B B} \cup J_{B 0} .
\end{gathered}
$$

Combining the last four equations with the first equation of (135), we obtain

$$
\begin{aligned}
&\langle\Delta \mu, \Delta \mu\rangle+2\langle\Delta y, \Delta y\rangle \\
&=\left\langle\mathscr{J}_{\zeta} h(\bar{\zeta}) d_{1}, \Delta \mu\right\rangle-\left\langle\mathscr{J}_{\zeta} g(\bar{\zeta}) d_{1}-d_{2}, \Delta y\right\rangle \\
&-\left\langle d_{2}+v, \Delta y\right\rangle \\
&=\left\langle d_{1}, \mathscr{J}_{\zeta} h(\bar{\zeta})^{T} \Delta \mu-\mathscr{J}_{\zeta} g(\bar{\zeta})^{T} \Delta y\right\rangle-\langle v, \Delta y\rangle \\
&=-\langle v, \Delta y\rangle=0 .
\end{aligned}
$$

Thus, $\Delta \mu=0$ and $\Delta y=0$. Along with $\Delta \zeta=0$, we show that $W$ is nonsingular.

Note that $-y \in \mathscr{N}_{\mathscr{K}}(x)$ if and only if $-x \in \mathcal{N}_{\mathscr{K}}(y)$. The KKT conditions in (7) can be equivalently written as the following generalized equation

$$
0 \in\left[\begin{array}{c}
\mathscr{J}_{\zeta, x} L(\zeta, x, \mu, s, y) \\
h(\zeta) \\
g(\zeta)-x \\
x
\end{array}\right]+\left[\begin{array}{c}
\mathcal{N}_{\mathbb{R}^{n} \times \mathbb{R}^{n}}(\zeta, x) \\
\mathcal{N}_{\mathbb{R}^{m}}(\mu) \\
\mathcal{N}_{\mathbb{R}^{n}}(s) \\
\mathcal{N}_{\mathscr{K}}(y)
\end{array}\right]
$$

which is clear in the form of the generalized equation given by (29). Now using Proposition 22 and [9, Theorem 3.1], we may establish the main result of this paper, which states that Clarke's Jacobian of $E_{\mathrm{FB}}$ at a KKT point is nonsingular if and only if the KKT point is a strongly regular solution to the generalized equation (141).
Theorem 23. Let $(\bar{\zeta}, \bar{x})$ be a locally optimal solution to the nonlinear SOCP (4). Suppose that Robinson's CQ holds at this point. Let $(\bar{\mu}, \bar{s}, \bar{y}) \in \mathbb{R}^{m} \times \mathbb{R}^{n} \times \mathscr{K}$ be such that $(\bar{\zeta}, \bar{x}, \bar{\mu}, \bar{s}, \bar{y})$ is a KKT point of (4). Then the following statements are equivalent.

(a) The strong second-order sufficient condition in Definition 21 holds at $(\bar{\zeta}, \bar{x})$ and $(\bar{\zeta}, \bar{x})$ is constraint nondegenerate.

(b) Any element in $\partial E_{\mathrm{FB}}(\bar{\zeta}, \bar{x}, \bar{\mu}, \bar{s}, \bar{y})$ is nonsingular.

(c) Any element in $\partial E_{\mathrm{NR}}(\bar{\zeta}, \bar{x}, \bar{\mu}, \bar{s}, \bar{y})$ is nonsingular.

(d) $(\bar{\zeta}, \bar{x}, \bar{\mu}, \bar{s}, \bar{y})$ is a strongly regular solution of the generalized equation (141).

Proof. First, Lemma 14 and the definition of $E_{\mathrm{FB}}$ and $E_{\mathrm{NR}}$ imply the following inclusion:

$$
\partial E_{\mathrm{NR}}(\bar{\zeta}, \bar{x}, \bar{\mu}, \bar{s}, \bar{y}) \subseteq \partial E_{\mathrm{FB}}(\bar{\zeta}, \bar{x}, \bar{\mu}, \bar{s}, \bar{y})
$$

Using this inclusion and Proposition 22, we have that (a) $\Rightarrow(\mathrm{b}) \Rightarrow$ (c). Since the SOCP (4) is obtained from (1) by introducing a slack variable, we know from [9, Theorem 3.1] that (a) $\Leftrightarrow$ (c) $\Leftrightarrow(\mathrm{d})$. Thus, we complete the proof of this theorem.

\section{Conclusions}

In this paper, for a locally optimal solution of the nonlinear SOCP, we established the equivalence between the nonsingularity of Clarke's Jacobian of the FB system and the strong regularity of the corresponding KKT point. This provides a new characterization for the strong regularity of the nonlinear SOCPs and extends the result of [22, Corollary 3.7] for the FB system of variational inequalities with the polyhedral cone $\mathbb{R}_{+}^{n}$ constraints to the setting of SOCs. Also, this result implies that the semismooth Newton method $[5,6]$ applied to the FB system is locally quadratically convergent to a KKT point under the strong second-order sufficient condition and constraint nondegeneracy. We point it out that we have also established parallel (not exactly the same) results for SDP case in [11] recently. However, it seems hard to put them together in a unified framework under Euclidean Jordan algebra. The main reason causing this is due to that the analysis and techniques are totally different when dealing with the Clarke Jacobians associated with FB SOC complementarity function and FB SDP complementarity function.

\section{Appendix}

Lemma A.1. For any given $x=\left(x_{1}, x_{2}\right), y=\left(y_{1}, y_{2}\right)$ with $x^{2}+y^{2} \in \mathbf{b d}^{+} \mathscr{K}^{n}$, it holds that

$$
\begin{gathered}
\partial_{B} \phi_{\mathrm{FB}}(x, y)=\partial_{B} \psi_{\mathrm{FB}}(0,0) \\
\text { with } \psi_{\mathrm{FB}}(\cdot, \cdot) \equiv \phi_{\mathrm{FB}}^{\prime}((x, y) ;(\cdot, \cdot)) .
\end{gathered}
$$

Proof. We first prove that $\partial_{B} \psi_{\mathrm{FB}}(0,0) \subseteq \partial_{B} \phi_{\mathrm{FB}}(x, y)$. Let $\left[\begin{array}{ll}U & V\end{array}\right] \in \partial_{B} \psi_{\mathrm{FB}}(0,0)$. By the formula (32) and Lemma 11, 
there exists a vector $\left(\xi_{1}, \xi_{2}\right) \in \mathbb{R} \times \mathbb{R}^{n-1}$ with $\xi_{1}^{2}+\left\|\xi_{2}\right\|^{2}=1$ such that $u$ and $v$ defined by (60) satisfy

$$
U=I-X-\frac{1}{2}\left(\begin{array}{c}
1 \\
-\widetilde{w}_{2}
\end{array}\right) u^{T}, \quad V=I-Y-\frac{1}{2}\left(\begin{array}{c}
1 \\
-\widetilde{w}_{2}
\end{array}\right) v^{T},
$$

where $X$ and $Y$ are defined by (64). Take the sequences $\left\{x^{k}\right\}$ and $\left\{y^{k}\right\}$ with

$$
x^{k} \equiv\left(\begin{array}{c}
x_{1}+\frac{1}{k} x_{1} \xi_{1} \\
x_{2}-\frac{1}{k} y_{1} \xi_{2}
\end{array}\right), \quad y^{k} \equiv\left(\begin{array}{c}
y_{1}+\frac{1}{k} y_{1} \xi_{1} \\
y_{2}+\frac{1}{k} x_{1} \xi_{2}
\end{array}\right) .
$$

Let $w^{k}=\left(w_{1}^{k}, w_{2}^{k}\right)=\left(x^{k}\right)^{2}+\left(y^{k}\right)^{2}$. By Lemma 5, a simple computation yields that

$$
\begin{gathered}
w_{2}^{k}=\left(1+\frac{\xi_{1}}{k}\right) w_{2}, \quad \lambda_{1}\left(w^{k}\right)=\frac{x_{1}^{2}+y_{1}^{2}}{k^{2}}, \\
\lambda_{2}\left(w^{k}\right)=\lambda_{2}(w)+O\left(\frac{1}{k}\right) .
\end{gathered}
$$

Clearly, $z^{k}=\sqrt{w^{k}} \in$ int $\mathscr{K}^{n}$. From Corollary 10(a), it then follows that

$$
\begin{aligned}
& \lim _{k \rightarrow \infty} \mathscr{J}_{x} \phi_{\mathrm{FB}}\left(x^{k}, y^{k}\right)=I-\lim _{k \rightarrow \infty} L_{z^{k}}^{-1} L_{x^{k}}, \\
& \lim _{k \rightarrow \infty} \mathcal{J}_{y} \phi_{\mathrm{FB}}\left(x^{k}, y^{k}\right)=I-\lim _{k \rightarrow \infty} L_{z^{k}}^{-1} L_{y^{k}} .
\end{aligned}
$$

Let $\widetilde{w}_{2}^{k}=w_{2}^{k} /\left\|w_{2}^{k}\right\|$. Using the formula (19), we have that $L_{z^{k}}^{-1}=$ $L\left(w_{1}^{k}\right)+L\left(w_{2}^{k}\right)$, where

$$
L_{1}\left(w^{k}\right):=\frac{1}{2 \sqrt{\lambda_{1}\left(w^{k}\right)}}\left(\begin{array}{cc}
1 & -\left(\widetilde{w}_{2}^{k}\right)^{T} \\
-\widetilde{w}_{2}^{k} & \widetilde{w}_{2}^{T}\left(\widetilde{w}_{2}^{k}\right)^{T}
\end{array}\right),
$$

$$
\begin{aligned}
L_{2}\left(w^{k}\right) & \\
:= & \frac{1}{2 \sqrt{\lambda_{2}\left(w^{k}\right)}} \\
& \times\left(\begin{array}{c}
1 \\
\left.\widetilde{w}_{2}^{k} \frac{4 \sqrt{\lambda_{2}\left(w^{k}\right)}\left(I-\widetilde{w}_{2}^{k}\left(\widetilde{w}_{2}^{k}\right)^{T}\right)}{\sqrt{\lambda_{2}\left(w^{k}\right)}+\sqrt{\lambda_{1}\left(w^{k}\right)}}+\widetilde{w}_{2}^{k}\left(\widetilde{w}_{2}^{k}\right)^{T}\right) .
\end{array}\right.
\end{aligned}
$$

Since $\lambda_{1}\left(w^{k}\right) \rightarrow 0, \lambda_{2}\left(w^{k}\right) \rightarrow 2 w_{1}>0$ and $\widetilde{w}_{2}^{k} \rightarrow \widetilde{w}_{2}$ as $k \rightarrow \infty$, it follows that

$$
\begin{aligned}
& \lim _{k \rightarrow \infty} L_{2}\left(w^{k}\right) L_{x^{k}}=\frac{1}{2 \sqrt{2 w_{1}}}\left(\begin{array}{cc}
1 & \widetilde{w}_{2}^{T} \\
\widetilde{w}_{2} & 4 I-3 \widetilde{w}_{2} \widetilde{w}_{2}^{T}
\end{array}\right) L_{x}=X, \\
& \lim _{k \rightarrow \infty} L_{2}\left(w^{k}\right) L_{y^{k}}=\frac{1}{2 \sqrt{2 w_{1}}}\left(\begin{array}{cc}
1 & \widetilde{w}_{2}^{T} \\
\widetilde{w}_{2} & 4 I-3 \widetilde{w}_{2} \widetilde{w}_{2}^{T}
\end{array}\right) L_{y}=Y,
\end{aligned}
$$

where the last two equalities are using Lemma 5. In addition, we compute that

$$
\begin{aligned}
& L_{1}\left(w^{k}\right) L_{x^{k}}=\frac{1}{2}\left(\begin{array}{cc}
u_{1}^{k} & u_{2}^{k} \\
-u_{1}^{k} \widetilde{w}_{2}^{k} & -\widetilde{w}_{2}^{k}\left(u_{2}^{k}\right)^{T}
\end{array}\right)=\left(\begin{array}{c}
1 \\
-\widetilde{w}_{2}^{k}
\end{array}\right)\left(u^{k}\right)^{T}, \\
& L_{1}\left(w^{k}\right) L_{y^{k}}=\frac{1}{2}\left(\begin{array}{cc}
v_{1}^{k} & v_{2}^{k} \\
-v_{1}^{k} \widetilde{w}_{2}^{k} & -\widetilde{w}_{2}^{k}\left(v_{2}^{k}\right)^{T}
\end{array}\right)=\left(\begin{array}{c}
1 \\
-\widetilde{w}_{2}^{k}
\end{array}\right)\left(v^{k}\right)^{T},
\end{aligned}
$$

where $u^{k}=\left(u_{1}^{k}, u_{2}^{k}\right), v^{k}=\left(v_{1}^{k}, v_{2}^{k}\right) \in \mathbb{R} \times \mathbb{R}^{n-1}$ are defined as follows:

$$
\begin{array}{ll}
u_{1}^{k}=\frac{x_{1}^{k}-\left(x_{2}^{k}\right)^{T} \widetilde{w}_{2}^{k}}{\sqrt{\lambda_{1}\left(w^{k}\right)}}, & u_{2}^{k}=\frac{x_{2}^{k}-x_{1}^{k} \widetilde{w}_{2}^{k}}{\sqrt{\lambda_{1}\left(w^{k}\right)}}, \\
v_{1}^{k}=\frac{y_{1}^{k}-\left(y_{2}^{k}\right)^{T} \widetilde{w}_{2}^{k}}{\sqrt{\lambda_{1}\left(w^{k}\right)}}, & v_{2}^{k}=\frac{y_{2}^{k}-y_{1}^{k} \widetilde{w}_{2}^{k}}{\sqrt{\lambda_{1}\left(w^{k}\right)}} .
\end{array}
$$

Together with the definition of $x^{k}$ and $y^{k}$ and (A.4), we can verify that

$$
\lim _{k \rightarrow \infty}\left(u_{1}^{k}, u_{2}^{k}\right)=u, \quad \lim _{k \rightarrow \infty}\left(v_{1}^{k}, v_{2}^{k}\right)=v .
$$

Thus, the above arguments show that

$$
\begin{aligned}
& \lim _{k \rightarrow \infty} \mathscr{J}_{x} \phi_{\mathrm{FB}}\left(x^{k}, y^{k}\right)=I-X-\frac{1}{2}\left(\begin{array}{c}
1 \\
-\widetilde{w}_{2}
\end{array}\right) u^{T} ; \\
& \lim _{k \rightarrow \infty} \mathscr{J}_{y} \phi_{\mathrm{FB}}\left(x^{k}, y^{k}\right)=I-Y-\frac{1}{2}\left(\begin{array}{c}
1 \\
-\widetilde{w}_{2}
\end{array}\right) v^{T} .
\end{aligned}
$$

Comparing this with (A.2), we have $\left[\begin{array}{ll}U & V\end{array}\right] \in \partial_{B} \phi_{\mathrm{FB}}(x, y)$. So, $\partial_{B} \psi_{\mathrm{FB}}(0,0) \subseteq \partial_{B} \phi_{\mathrm{FB}}(x, y)$.

In what follows, we show that $\partial_{B} \phi_{\mathrm{FB}}(x, y) \subseteq \partial_{B} \psi_{\mathrm{FB}}(0,0)$. Note that $x^{2}+y^{2} \in \mathbf{b d}^{+} \mathscr{K}^{n}$ is equivalent to $(x, y) \neq(0,0)$ and $\|x\|^{2}+\|y\|^{2}=2\left\|x_{1} x_{2}+y_{1} y_{2}\right\|$, which is equivalent to

$$
\begin{aligned}
\left(\left|x_{1}\right|\right. & \left.-\left\|x_{2}\right\|\right)^{2}+\left(\left|y_{1}\right|-\left\|y_{2}\right\|\right)^{2} \\
& +2\left(\left|x_{1}\right|\left\|x_{2}\right\|+\left|y_{1}\right|\left\|y_{2}\right\|-\left\|x_{1} x_{2}+y_{1} y_{2}\right\|\right)=0 .
\end{aligned}
$$

Hence, $x^{2}+y^{2} \in \mathbf{b d}^{+} \mathscr{K}^{n}$ is equivalent to saying that $x=$ $\left(x_{1}, x_{2}\right), y=\left(y_{1}, y_{2}\right)$ satisfy

$$
\begin{gathered}
\left|x_{1}\right|=\left\|x_{2}\right\|, \quad\left|y_{1}\right|=\left\|y_{2}\right\|, \\
x_{1} y_{1} x_{2}^{T} y_{2}=\left|x_{1}\right|\left|y_{1}\right|\left\|x_{2}\right\|\left\|y_{2}\right\|, \quad(x, y) \neq(0,0) .
\end{gathered}
$$

This means that $x, y$ must satisfy one of the following cases: (i) $x=\alpha y$ for some $\alpha \in \mathbb{R}$ and $y^{2} \in \mathbf{b d}^{+} \mathscr{K}^{n}$; (ii) $y=\beta x$ 
for some $\beta \in \mathbb{R}$ and $x^{2} \in \mathbf{b d}^{+} \mathscr{K}^{n}$. Since $\phi_{\mathrm{FB}}$ and $\psi_{\mathrm{FB}}$ are symmetric with respect to two arguments, we only need to prove one of the two cases. In the following arguments, we assume that $x=\alpha y$ for some $\alpha \in \mathbb{R}$ and $y^{2} \in \mathbf{b d}^{+} \mathscr{K}^{n}$. Noting that $y_{1} \neq 0$ since $y^{2} \in \mathbf{b d}^{+} \mathscr{K}^{n}$, we without loss of generality assume that $y_{1}>0$. From (32) and Lemma 11, it is not hard to see that

$$
\begin{aligned}
& \partial_{B} \psi_{\mathrm{FB}}(0,0) \\
& =\left\{\left[I-X-\frac{1}{2}\left(\begin{array}{c}
1 \\
-\widetilde{w}_{2}
\end{array}\right) u^{T} I-Y-\frac{1}{2}\left(\begin{array}{c}
1 \\
-\widetilde{w}_{2}
\end{array}\right) v^{T}\right] \mid\right. \\
& \text { for some } u=\left(\frac{\xi_{2}^{T} \tilde{y}_{2}+\alpha \xi_{1}}{\sqrt{1+\alpha^{2}}}, \frac{-\xi_{2}-\alpha \xi_{1} \widetilde{y}_{2}}{\sqrt{1+\alpha^{2}}}\right), \\
& v=\left(\frac{-\alpha \xi_{2}^{T} \widetilde{y}_{2}+\xi_{1}}{\sqrt{1+\alpha^{2}}}, \frac{\alpha \xi_{2}-\xi_{1} \tilde{y}_{2}}{\sqrt{1+\alpha^{2}}}\right)
\end{aligned}
$$

with $\xi=\left(\xi_{1}, \xi_{2}\right)$ satisfying $\xi_{1}^{2}+\left\|\xi_{2}\right\|^{2}=1$,

where $\tilde{y}_{2}=\frac{y_{2}}{\left\|y_{2}\right\|}$ and

the $n \times n$ symmetric matrices $X$ and $Y$

are defined by (64) $\}$.

Let $\left[\begin{array}{ll}U & V\end{array}\right] \in \partial_{B} \phi_{\mathrm{FB}}(x, y)$. By the definition of the elements in $\partial_{B} \phi_{\mathrm{FB}}(x, y)$, there exists a sequence $\left\{\left(x^{k}, y^{k}\right)\right\}$ with $w^{k}=$ $\left(x^{k}\right)^{2}+\left(y^{k}\right)^{2} \in$ int $\mathscr{K}^{n}$ converging to $(x, y)$ such that $[U V]=$ $\lim _{k \rightarrow \infty} \mathscr{J} \phi_{\mathrm{FB}}\left(x^{k}, y^{k}\right)$. From the arguments for the first part, we know that

$$
\begin{aligned}
& U=I-X-\lim _{k \rightarrow \infty} \frac{1}{2}\left(\begin{array}{c}
1 \\
-\widetilde{w}_{2}
\end{array}\right)\left(u_{1}^{k},\left(u_{2}^{k}\right)^{T}\right), \\
& V=I-Y-\lim _{k \rightarrow \infty} \frac{1}{2}\left(\begin{array}{c}
1 \\
-\widetilde{w}_{2}
\end{array}\right)\left(v_{1}^{k},\left(v_{2}^{k}\right)^{T}\right),
\end{aligned}
$$

with $u^{k}=\left(u_{1}^{k}, u_{2}^{k}\right)$ and $v^{k}=\left(v_{1}^{k}, v_{2}^{k}\right)$ defined by (A.9). Thus, in order to prove that $\left[\begin{array}{ll}U & V\end{array}\right] \in \partial_{B} \psi_{\mathrm{FB}}(0,0)$, it suffices to argue that the following limits:

$$
\begin{aligned}
& \lim _{k \rightarrow \infty}\left(u_{1}^{k}, u_{2}^{k}\right)=\left(\frac{\bar{\xi}_{2}^{T} \tilde{y}_{2}+\alpha \bar{\xi}_{1}}{\sqrt{1+\alpha^{2}}}, \frac{-\bar{\xi}_{2}-\alpha \bar{\xi}_{1} \tilde{y}_{2}}{\sqrt{1+\alpha^{2}}}\right), \\
& \lim _{k \rightarrow \infty}\left(v_{1}^{k}, v_{2}^{k}\right)=\left(\frac{-\alpha \bar{\xi}_{2}^{T} \tilde{y}_{2}+\bar{\xi}_{1}}{\sqrt{1+\alpha^{2}}}, \frac{\alpha \bar{\xi}_{2}-\bar{\xi}_{1} \tilde{y}_{2}}{\sqrt{1+\alpha^{2}}}\right),
\end{aligned}
$$

hold for some $\bar{\xi}=\left(\bar{\xi}_{1}, \bar{\xi}_{2}\right)$ with $\bar{\xi}_{1}^{2}+\left\|\bar{\xi}_{2}\right\|^{2}=1$. We proceed the arguments by two steps.

Step 1. To prove $\lim _{k \rightarrow \infty}\left(\left(\left(\alpha u_{1}^{k} v_{1}^{k}\right)^{2}+\left\|\alpha v_{2}^{k}-u_{2}^{k}\right\|^{2}\right) /\left(1+\alpha^{2}\right)\right)=$ 1 (taking a subsequence if necessary). For each $k$, by the expressions of $u_{1}^{k}, u_{2}^{k}$, and $v_{1}^{k}, v_{2}^{k}$, it is easy to see that

$$
\begin{aligned}
\frac{\left(\alpha u_{1}^{k}+v_{1}^{k}\right)^{2}+\left\|\alpha v_{2}^{k}-u_{2}^{k}\right\|^{2}}{1+\alpha^{2}} & \\
= & \frac{\left[\left(\alpha x_{1}^{k}+y_{1}^{k}\right)-\left(\alpha x_{2}^{k}+y_{2}^{k}\right)^{T} \widetilde{w}_{2}^{k}\right]^{2}}{\left(1+\alpha^{2}\right) \lambda_{1}\left(w^{k}\right)} \\
+ & \frac{\left\|\left(\alpha y_{2}^{k}-x_{2}^{k}\right)-\left(\alpha y_{1}^{k}-x_{1}^{k}\right) \widetilde{w}_{2}^{k}\right\|^{2}}{\left(1+\alpha^{2}\right) \lambda_{1}\left(w^{k}\right)}
\end{aligned}
$$

where $\widetilde{w}_{2}^{k}=w_{2}^{k} /\left\|w_{2}\right\|$ and $w_{2}^{k}=2\left\|x_{1}^{k} x_{2}^{k}+y_{1}^{k} y_{2}^{k}\right\|$. An elementary computation yields that

$$
\begin{aligned}
{\left[\left(\alpha x_{1}^{k}+y_{1}^{k}\right)-\left(\alpha x_{2}^{k}+y_{2}^{k}\right)^{T} \widetilde{w}_{2}^{k}\right]^{2} } & \\
+ & \left\|\left(\alpha y_{2}^{k}-x_{2}^{k}\right)-\left(\alpha y_{1}^{k}-x_{1}^{k}\right) \widetilde{w}_{2}^{k}\right\|^{2} \\
= & \left(1+\alpha^{2}\right) \lambda_{1}\left(w^{k}\right)-\left\|y_{2}^{k}+\alpha x_{2}^{k}\right\|^{2} \\
+ & \frac{\left[\left(\alpha x_{2}^{k}+y_{2}^{k}\right)^{T}\left(x_{1}^{k} x_{2}^{k}+y_{1}^{k} y_{2}^{k}\right)\right]^{2}}{\left\|x_{1}^{k} x_{2}^{k}+y_{1}^{k} y_{2}^{k}\right\|^{2}} .
\end{aligned}
$$

From the last two equations, we immediately obtain that

$$
\begin{aligned}
\frac{\left(\alpha u_{1}^{k}+v_{1}^{k}\right)^{2}+\left\|\alpha v_{2}^{k}-u_{2}^{k}\right\|^{2}}{1+\alpha^{2}} \\
=1-\frac{\left\|y_{2}^{k}+\alpha x_{2}^{k}\right\|^{2}}{\left(1+\alpha^{2}\right) \lambda_{1}\left(w^{k}\right)} \\
+\frac{\left[\left(\alpha x_{2}^{k}+y_{2}^{k}\right)^{T}\left(x_{1}^{k} x_{2}^{k}+y_{1}^{k} y_{2}^{k}\right)\right]^{2}}{\left(1+\alpha^{2}\right) \lambda_{1}\left(w^{k}\right)\left\|x_{1}^{k} x_{2}^{k}+y_{1}^{k} y_{2}^{k}\right\|^{2}} .
\end{aligned}
$$

This shows that, in order to achieve the result, it suffices to prove that

$$
\lim _{k \rightarrow \infty}\left[\frac{\left\|y_{2}^{k}+\alpha x_{2}^{k}\right\|^{2}}{\lambda_{1}\left(w^{k}\right)}-\frac{\left[\left(\alpha x_{2}^{k}+y_{2}^{k}\right)^{T}\left(x_{1}^{k} x_{2}^{k}+y_{1}^{k} y_{2}^{k}\right)\right]^{2}}{\lambda_{1}\left(w^{k}\right)\left\|x_{1}^{k} x_{2}^{k}+y_{1}^{k} y_{2}^{k}\right\|^{2}}\right]=0,
$$

which is equivalent to arguing that

$$
\lim _{k \rightarrow \infty} \frac{Q\left(x^{k}, y^{k}\right)}{\lambda_{1}\left(w^{k}\right)\left\|x_{1}^{k} x_{2}^{k}+y_{1}^{k} y_{2}^{k}\right\|^{2}}=0
$$


with

$$
\begin{aligned}
Q\left(x^{k}, y^{k}\right):= & \left\|y_{2}^{k}+\alpha x_{2}^{k}\right\|^{2}\left\|x_{1}^{k} x_{2}^{k}+y_{1}^{k} y_{2}^{k}\right\|^{2} \\
& -\left[\left(\alpha x_{2}^{k}+y_{2}^{k}\right)^{T}\left(x_{1}^{k} x_{2}^{k}+y_{1}^{k} y_{2}^{k}\right)\right]^{2} .
\end{aligned}
$$

An elementary computation yields that

$$
\begin{gathered}
\frac{Q\left(x^{k}, y^{k}\right)}{\lambda_{1}\left(w^{k}\right)\left\|x_{1}^{k} x_{2}^{k}+y_{1}^{k} y_{2}^{k}\right\|^{2}} \\
=\frac{\left(x_{1}^{k}-\alpha y_{1}^{k}\right)^{2}\left[\left\|x_{2}^{k}\right\|^{2}\left\|y_{2}^{k}\right\|^{2}-\left(\left(x_{2}^{k}\right)^{T} y_{2}^{k}\right)^{2}\right]}{\lambda_{1}\left(w^{k}\right)\left\|x_{1}^{k} x_{2}^{k}+y_{1}^{k} y_{2}^{k}\right\|^{2}} \\
=\frac{\left(x_{1}^{k}-\alpha y_{1}^{k}\right)^{2}\left[\left\|x_{2}^{k}\right\|^{2}\left\|y_{2}^{k}\right\|^{2}-\left(\left(x_{2}^{k}\right)^{T} y_{2}^{k}\right)^{2}\right] \lambda_{2}\left(w^{k}\right)}{\left\|x_{1}^{k} x_{2}^{k}+y_{1}^{k} y_{2}^{k}\right\|^{2}\left[\left(w_{1}^{k}\right)^{2}-\left\|w_{2}^{k}\right\|^{2}\right]} .
\end{gathered}
$$

By the expressions of $w_{1}^{k}$ and $w_{2}^{k}$, we compute that

$$
\begin{aligned}
\left(w_{1}^{k}\right)^{2}-\left\|w_{2}^{k}\right\|^{2}= & \left(\left(x_{1}^{k}\right)^{2}-\left\|x_{2}^{k}\right\|^{2}\right)^{2}+\left(\left(y_{1}^{k}\right)^{2}-\left\|y_{2}^{k}\right\|^{2}\right)^{2} \\
& +2\left(\left(x_{1}^{k}\right)^{2}+\left\|x_{2}^{k}\right\|^{2}\right)\left(\left(y_{1}^{k}\right)^{2}+\left\|y_{2}^{k}\right\|^{2}\right) \\
& -8 x_{1}^{k} y_{1}^{k}\left(x_{2}^{k}\right)^{T} y_{2}^{k} .
\end{aligned}
$$

Substep $1.1(\alpha>0)$. Since $y_{1}>0$ and $x_{1}=\alpha y_{1}>0$, we have $x_{1}^{k} y_{1}^{k}>0$ for sufficiently large $k$. In addition, from (A.24), it is not difficult to obtain that

$$
\begin{aligned}
\left(w_{1}^{k}\right)^{2}-\left\|w_{2}^{k}\right\|^{2}= & \left(\left(x_{1}^{k}\right)^{2}-\left\|x_{2}^{k}\right\|^{2}\right)^{2}+\left(\left(y_{1}^{k}\right)^{2}-\left\|y_{2}^{k}\right\|^{2}\right)^{2} \\
& +2\left(x_{1}^{k} y_{1}^{k}-\left\|x_{2}^{k}\right\|\left\|y_{2}^{k}\right\|\right)^{2} \\
& +2\left\|x_{1}^{k} y_{2}^{k}-y_{1}^{k} x_{2}^{k}\right\|^{2} \\
& +8 x_{1}^{k} y_{1}^{k}\left[\left\|x_{2}^{k}\right\|\left\|y_{2}^{k}\right\|-\left(x_{2}^{k}\right)^{T} y_{2}^{k}\right] \\
\geq & 8 x_{1}^{k} y_{1}^{k}\left[\left\|x_{2}^{k}\right\|\left\|y_{2}^{k}\right\|-\left(x_{2}^{k}\right)^{T} y_{2}^{k}\right] .
\end{aligned}
$$

Together with (A.23) and $x_{1}^{k} y_{1}^{k}>0$, we have that

$$
\begin{aligned}
0 & \leq \frac{Q\left(x^{k}, y^{k}\right)}{\lambda_{1}\left(w^{k}\right)\left\|x_{1}^{k} x_{2}^{k}+y_{1}^{k} y_{2}^{k}\right\|^{2}} \\
& \leq \frac{\left(x_{1}^{k}-\alpha y_{1}^{k}\right)^{2}\left[\left\|x_{2}^{k}\right\|\left\|y_{2}^{k}\right\|+\left(x_{2}^{k}\right)^{T} y_{2}^{k}\right] \lambda_{2}\left(w^{k}\right)}{8 x_{1}^{k} y_{1}^{k}\left\|x_{1}^{k} x_{2}^{k}+y_{1}^{k} y_{2}^{k}\right\|^{2}} .
\end{aligned}
$$

Taking the limit $k \rightarrow \infty$ to the inequality, we obtain the limit in (A.21).

Substep $1.2(\alpha<0)$. Since $y_{1}>0$ and $x_{1}=\alpha y_{1}<0$, we have $x_{1}^{k} y_{1}^{k}<0$ for sufficiently large $k$. Now, from (A.24), it is easy to obtain that

$$
\begin{aligned}
\left(w_{1}^{k}\right)^{2}-\left\|w_{2}^{k}\right\|^{2}= & \left(\left(x_{1}^{k}\right)^{2}-\left\|x_{2}^{k}\right\|^{2}\right)^{2}+\left(\left(y_{1}^{k}\right)^{2}-\left\|y_{2}^{k}\right\|^{2}\right)^{2} \\
& +2\left(x_{1}^{k} y_{1}^{k}+\left\|x_{2}^{k}\right\|\left\|y_{2}^{k}\right\|\right)^{2} \\
& +2\left\|x_{1}^{k} y_{2}^{k}+y_{1}^{k} x_{2}^{k}\right\|^{2} \\
& -8 x_{1}^{k} y_{1}^{k}\left[\left\|x_{2}^{k}\right\|\left\|y_{2}^{k}\right\|+\left(x_{2}^{k}\right)^{T} y_{2}^{k}\right] \\
\geq & -8 x_{1}^{k} y_{1}^{k}\left[\left\|x_{2}^{k}\right\|\left\|y_{2}^{k}\right\|+\left(x_{2}^{k}\right)^{T} y_{2}^{k}\right] .
\end{aligned}
$$

Together with (A.23) and $x_{1}^{k} y_{1}^{k}<0$, it follows that

$$
\begin{aligned}
0 & \leq \frac{Q\left(x^{k}, y^{k}\right)}{\lambda_{1}\left(w^{k}\right)\left\|x_{1}^{k} x_{2}^{k}+y_{1}^{k} y_{2}^{k}\right\|^{2}} \\
& \leq \frac{\left(x_{1}^{k}-\alpha y_{1}^{k}\right)^{2}\left[\left\|x_{2}^{k}\right\|\left\|y_{2}^{k}\right\|-\left(x_{2}^{k}\right)^{T} y_{2}^{k}\right] \lambda_{2}\left(w^{k}\right)}{-8 x_{1}^{k} y_{1}^{k}\left\|x_{1}^{k} x_{2}^{k}+y_{1}^{k} y_{2}^{k}\right\|^{2}} .
\end{aligned}
$$

Taking the limit $k \rightarrow \infty$ to this inequality, we readily obtain the limit in (A.21).

Substep $1.3(\alpha=0)$. Now we must have $x_{1}^{k}>0$ or $x_{1}^{k}<0$ for sufficiently large $k$. Then, using the same arguments as in Substepss 1.1 and 1.2, we get the limit in (A.21).

Let $u=\left(u_{1}, u_{2}\right)=\lim _{k \rightarrow \infty} u^{k}$ and $v=\left(v_{1}, v_{2}\right)=$ $\lim _{k \rightarrow \infty} v^{k}$ with $u^{k}$ and $v^{k}$ defined by (A.9).

Step 2. To prove that $\alpha u_{2}+\alpha u_{1} \tilde{y}_{2}+v_{2}+v_{1} \tilde{y}_{2}=0$. By the expression of $u^{k}$ and $v^{k}$, it is easy to verify that $\left(u_{2}^{k}\right)^{T} \widetilde{w}_{2}^{k}=-u_{1}^{k}$ and $\left(v_{2}^{k}\right)^{T} \widetilde{w}_{2}^{k}=-v_{1}^{k}$, which implies that

$$
u_{2}^{T} \tilde{y}_{2}=-u_{1}, \quad v_{2}^{T} \tilde{y}_{2}=-v_{1} .
$$

By (A.29), we can verify that $\left\|\alpha u_{2}+\alpha u_{1} \tilde{y}_{2}+v_{2}+v_{1} \tilde{y}_{2}\right\|=0$ is equivalent to $\left\|\alpha u_{2}+v_{2}\right\|^{2}=\left(\alpha u_{1}+v_{1}\right)^{2}$. From Step 1, we have that $\left(\alpha u_{1}+v_{1}\right)^{2}+\left\|\alpha v_{2}-u_{2}\right\|^{2}=1+\alpha^{2}$. This implies that, to prove that $\alpha u_{2}+\alpha u_{1} \tilde{y}_{2}+v_{2}+v_{1} \tilde{y}_{2}=0$, it suffices to argue that

$$
\left\|u_{2}\right\|^{2}+\left\|v_{2}\right\|^{2}=1
$$

Indeed, noting that $\left\|x_{2}^{k}-x_{1}^{k} \widetilde{w}_{2}\right\|^{2}+\left\|y_{2}^{k}-y_{1}^{k} \widetilde{w}_{2}^{k}\right\|^{2}=\lambda_{1}\left(w^{k}\right)$, we readily have that

$$
\left\|u_{2}\right\|^{2}+\left\|v_{2}\right\|^{2}=\lim _{k \rightarrow \infty} \frac{\left\|x_{2}^{k}-x_{1}^{k} \widetilde{w}_{2}\right\|^{2}}{\lambda_{1}\left(w^{k}\right)}+\frac{\left\|y_{2}^{k}-y_{1}^{k} \widetilde{w}_{2}^{k}\right\|^{2}}{\lambda\left(w^{k}\right)}=1 .
$$


Now let $\bar{\xi}_{1}=\left(\alpha u_{1}+v_{1}\right) / \sqrt{1+\alpha^{2}}$ and $\bar{\xi}_{2}=\left(\alpha v_{2}-u_{2}\right) /$ $\sqrt{1+\alpha^{2}}$. Clearly, $\bar{\xi}_{1}^{2}+\left\|\bar{\xi}_{2}\right\|^{2}=1$. Also, using the results of Steps 1 and 2 and (A.29), we can verify that

$$
\begin{array}{ll}
u_{1}=\frac{\bar{\xi}_{2}^{T} \tilde{y}_{2}+\alpha \bar{\xi}_{1}}{\sqrt{1+\alpha^{2}}}, & u_{2}=\frac{-\bar{\xi}_{2}-\alpha \bar{\xi}_{1} \tilde{y}_{2}}{\sqrt{1+\alpha^{2}}}, \\
v_{1}=\frac{-\alpha \bar{\xi}_{2}^{T} \tilde{y}_{2}+\bar{\xi}_{1}}{\sqrt{1+\alpha^{2}}}, & v_{2}=\frac{\alpha \bar{\xi}_{2}-\bar{\xi}_{1} \tilde{y}_{2}}{\sqrt{1+\alpha^{2}}} .
\end{array}
$$

This, along with (A.15), implied that $\left[\begin{array}{ll}U & V\end{array}\right] \in \partial_{B} \psi_{\mathrm{FB}}(0,0)$. The result then follows.

Proof of Lemma 15. Let $\widetilde{w}_{j}=\bar{w}_{j} /\left\|\bar{w}_{j}\right\|$ with $\bar{w}_{j}=\left(\bar{x}_{j}^{2}+\bar{y}_{j}^{2}\right)$ for $j=1, \ldots, r$.

(a) Since $\bar{x}_{j} \in \mathbf{b d}^{+} \mathscr{K}^{n_{j}}$ and $\bar{y}_{j}=0$, we have $\widetilde{w}_{j 2}=\widetilde{x}_{j 2}$. By Proposition 13 , there exist some $u_{j}=\left(u_{j 1}, u_{j 2}\right) \in \mathbb{R} \times \mathbb{R}^{n_{j}-1}$ and $v_{j}=\left(v_{j 1}, v_{j 2}\right) \in \mathbb{R} \times \mathbb{R}^{n_{j}-1}$ such that

$$
\begin{gathered}
U_{j}=I-\frac{1}{2}\left(\begin{array}{cc}
1 & \tilde{x}_{j 2}^{T} \\
\tilde{x}_{j 2} & 2 I-\widetilde{x}_{j 2} \widetilde{x}_{j 2}^{T}
\end{array}\right)-\frac{1}{\sqrt{2}} q_{j}^{\prime} u_{j}^{T}, \\
V_{j}=I-\frac{1}{\sqrt{2}} q_{j}^{\prime} v_{j}^{T},
\end{gathered}
$$

where

$$
\begin{gathered}
\left|u_{j 1}\right| \leq\left\|u_{j 2}\right\| \leq 1, \quad\left|v_{j 1}\right| \leq\left\|v_{j 2}\right\| \leq 1, \\
\left(u_{j 1}-v_{j 1}\right) \leq\left\|u_{j 2}-v_{j 2}\right\|, \\
\left(u_{j 1}+v_{j 1}\right) \leq\left\|u_{j 2}+v_{j 2}\right\|, \\
\left(u_{j 1}-v_{j 1}\right)^{2}+\left\|u_{j 2}+v_{j 2}\right\|^{2} \leq 2, \\
\left(u_{j 1}+v_{j 1}\right)^{2}+\left\|u_{j 2}-v_{j 2}\right\|^{2} \leq 2, \\
q_{j}^{T} u_{j}=0, \quad q_{j}^{T} v_{j}=0, \\
\left(q_{j}^{\prime}\right)^{T} u_{j}=\sqrt{2} u_{j 1}, \quad\left(q_{j}^{\prime}\right)^{T} v_{j}=\sqrt{2} v_{j 1} .
\end{gathered}
$$

It is not hard to verify that the matrix $I-(1 / 2)\left(\begin{array}{cc}1 & \widetilde{x}_{j 2}^{T} \\ \tilde{x}_{j 2} & 2 I-\tilde{x}_{j 2} \tilde{x}_{j 2}^{T}\end{array}\right)$ has eigenvalue 0 of multiplicity $n_{j}-1$ and a single eigenvalue 1 , with the corresponding eigenvectors being $q_{j}, \widehat{q}_{j}=\left(\begin{array}{c}0 \\ \hat{v}_{j}\end{array}\right)$ for $j=1, \ldots, n_{j}-2$, and $q_{j}^{\prime}$, respectively, where $\widehat{v}_{1}, \ldots, \widehat{v}_{n_{j}-2}$ are any unit vectors that span the linear subspace $\left\{\widehat{v} \in \mathbb{R}^{n_{j}-1}\right.$ | $\left.\widehat{v}^{T} \widetilde{x}_{j 2}=0\right\}$. Let $Q_{j}=\left[\begin{array}{lll}q_{j} & \widehat{q}_{1} \cdots \widehat{q}_{n_{j-2}} q_{j}^{\prime}\end{array}\right]$. Then such $Q_{j}$ is an $n_{j} \times n_{j}$ orthogonal matrix satisfying

$$
I-\frac{1}{2}\left(\begin{array}{cc}
1 & \tilde{x}_{j 2}^{T} \\
\tilde{x}_{j 2} & 2 I-\widetilde{x}_{j 2} \tilde{x}_{j 2}^{T}
\end{array}\right)=Q_{j} \operatorname{diag}(0,0, \ldots, 0,1) Q_{j}^{T} .
$$

Together with (A.33), we obtain $U_{j}=Q_{j} D_{j} Q_{j}^{T}$ and $V_{j}=$ $Q_{j} \Lambda_{j} Q_{j}^{T}$, where

$$
\begin{gathered}
D_{j}=\operatorname{diag}(0,0, \ldots, 0,1)-\frac{1}{\sqrt{2}} Q_{j}^{T} q_{j}^{\prime} u_{j}^{T} Q_{j}, \\
\Lambda_{j}=I-\frac{1}{\sqrt{2}} Q_{j}^{T} q_{j}^{\prime} v_{j}^{T} Q_{j} .
\end{gathered}
$$

Using the equalities in (A.34) yields $u_{j}^{T} Q_{j}=\left(0, u_{j}^{T} \widehat{Q}_{j}, \sqrt{2} u_{j 1}\right)$, and $v_{j}^{T} Q_{j}=\left(0, v_{j}^{T} \widehat{Q}_{j}, \sqrt{2} v_{j 1}\right)$ with $\widehat{Q}_{j}=\left[\widehat{q}_{1} \cdots \widehat{q}_{n_{j-2}}\right] \epsilon$ $\mathbb{R}^{n_{j} \times\left(n_{j}-2\right)}$. Along with $Q_{j}^{T} q_{j}^{\prime}=(0,0, \ldots, 0,1)^{T}$, we have that

$$
\begin{gathered}
D_{j}=\left(\begin{array}{ccc}
0 & 0 & 0 \\
0 & 0 & 0 \\
0 & -\frac{1}{\sqrt{2}} u_{j}^{T} \widehat{Q}_{j} & 1-u_{j 1}
\end{array}\right), \\
\Lambda_{j}=\left(\begin{array}{ccc}
1 & 0 & 0 \\
0 & I & 0 \\
0 & -\frac{1}{\sqrt{2}} v_{j}^{T} \widehat{Q}_{j} & 1-v_{j 1}
\end{array}\right) .
\end{gathered}
$$

Since $\left|u_{j 1}\right| \leq\left\|u_{j 2}\right\| \leq 1,\left|v_{j 1}\right| \leq\left\|v_{j 2}\right\| \leq 1$ and $\mid u_{j 1}+$ $v_{j 1} \mid \leq \sqrt{2}$, there are exactly three cases for the vectors $u_{j}$ and $v_{j}$ satisfying (A.34): (1) $u_{j 1}=1, v_{j 1}<1$; (2) $u_{j 1}<1, v_{j 1}=1$; (3) $u_{j 1}<1, v_{j 1}<1$. We next proceed the arguments by the three cases.

Case $1\left(u_{j 1}=1, v_{j 1}<1\right)$. Now we have $\left\|u_{j}\right\|=\sqrt{2}$. From the equality $\left(q_{j}^{\prime}\right)^{T} u_{j}=\sqrt{2}$ in (A.34) and $\left\|q_{j}^{\prime}\right\|=1$, we deduce that $u_{j}=\sqrt{2} q_{j}^{\prime}$, and hence $u_{j}^{T} \widehat{Q}_{j}=0$. In addition, from the last two inequalities of (A.34), $\left\|u_{j}\right\|^{2}+\left\|v_{j}\right\|^{2} \leq 2$, which together with $\left\|u_{j}\right\|^{2}=2$ implies $v_{j}=0$. Now plugging $u_{j}^{T} \widehat{Q}_{j}=0, u_{j 1}=$ $1, v_{j}=0$ into (A.37) yields $D_{j}=0$ and $\Lambda_{j}=I$. Therefore, $Q_{j}$ can be taken as an identity matrix.

Case $2\left(u_{j 1}<1, v_{j 1}=1\right)$. Under this case, since $\left(q_{j}^{\prime}\right)^{T} v_{j}=$ $\sqrt{2},\left\|v_{j}\right\|=\sqrt{2}$ and $\left\|q_{j}^{\prime}\right\|=1$, using the same arguments as in Case (1) then yields $v_{j}^{T} \widehat{Q}_{j}=0$ and $u_{j}=0$. Now plugging $v_{j}^{T} \widehat{Q}_{j}=0, v_{j 1}=1, u_{j}=0$ into (A.37), $D_{j}$ and $\Lambda_{j}$ become the one given by (85).

Case $3\left(u_{j 1}<1, v_{j 1}<1\right)$. By the expressions of $D_{j}$ and $\Lambda_{j}$, we calculate that

$$
\begin{aligned}
D_{j} \Lambda_{j}^{T}+\Lambda_{j} D_{j}^{T} & \\
& =\left(\begin{array}{ccc}
0 & 0 & 0 \\
0 & 0 & -\frac{1}{\sqrt{2}} \widehat{Q}_{j}^{T} u_{j} \\
0 & -\frac{1}{\sqrt{2}} u_{j}^{T} \widehat{Q}_{j} & 2\left(1-u_{j 1}\right)\left(1-v_{j 1}\right)+u_{j}^{T} \widehat{Q}_{j} \widehat{Q}_{j}^{T} v_{j}
\end{array}\right) .
\end{aligned}
$$

Since $\left[U_{j} V_{j}\right] \in \partial \phi_{\mathrm{FB}}\left(\bar{x}_{j}, \bar{y}_{j}\right)$, the definition of the elements in $\partial \phi_{\mathrm{FB}}\left(\bar{x}_{j}, \bar{y}_{j}\right)$ and the proof of [3, Lemma 6(b)] imply that 
$U_{j} V_{j}^{T}+V_{j} U_{j}^{T} \geq 0$, and hence $D_{j} \Lambda_{j}^{T}+\Lambda_{j} D_{j}^{T} \geq 0$. Thus, the zero diagonals imply $u_{j}^{T} \widehat{Q}_{j}=0$, and $D_{j}$ and $\Lambda_{j}$ have the expression of (86).

(b) In view of the symmetry of $\bar{x}$ and $\bar{y}$ in $U_{j}$ and $V_{j}$, the results readily follow by using similar arguments as in part (a). Thus, we complete the proof.

\section{Acknowledgments}

This work was supported by National Young Natural Science Foundation (no. 10901058) and Guangdong Natural Science Foundation (no. 9251802902000001), the Fundamental Research Funds for the Central Universities, and Project of Liaoning Innovative Research Team in University WT2010004. The author's work is supported by National Science Council of Taiwan, Department of Mathematics, National Taiwan Normal University, Taipei, Taiwan 11677.

\section{References}

[1] R. T. Rockafellar, Convex Analysis, Princeton University Press, Princeton, NJ, USA, 1970.

[2] D. F. Sun and J. Sun, "Strong semismoothness of the FischerBurmeister SDC and SOC complementarity functions," Mathematical Programming, vol. 103, no. 3, pp. 575-581, 2005.

[3] J.-S. Chen and P. Tseng, "An unconstrained smooth minimization reformulation of the second-order cone complementarity problem," Mathematical Programming, vol. 104, no. 2-3, pp. 293327, 2005.

[4] J.-S. Chen, D. F. Sun, and J. Sun, "The $S C^{1}$ property of the squared norm of the SOC Fischer-Burmeister function," Operations Research Letters, vol. 36, no. 3, pp. 385-392, 2008.

[5] J.-S. Pang and L. Q. Qi, "Nonsmooth equations: motivation and algorithms," SIAM Journal on Optimization, vol. 3, no. 3, pp. 443-465, 1993.

[6] L. Q. Qi and J. Sun, "A nonsmooth version of Newton's method," Mathematical Programming, vol. 58, no. 3, pp. 353-367, 1993.

[7] J.-F. Bonnans and H. C. Ramírez, "Perturbation analysis of second-order cone programming problems," Mathematical Programming, vol. 104, no. 2-3, pp. 205-227, 2005.

[8] Z. X. Chan and D. F. Sun, "Constraint nondegeneracy, strong regularity, and nonsingularity in semidefinite programming," SIAM Journal on Optimization, vol. 19, no. 1, pp. 370-396, 2008.

[9] Y. Wang and L. W. Zhang, "Properties of equation reformulation of the Karush-Kuhn-Tucker condition for nonlinear second order cone optimization problems," Mathematical Methods of Operations Research, vol. 70, no. 2, pp. 195-218, 2009.

[10] C. Kanzow, I. Ferenczi, and M. Fukushima, "On the local convergence of semismooth Newton methods for linear and nonlinear second-order cone programs without strict complementarity," SIAM Journal on Optimization, vol. 20, no. 1, pp. 297-320, 2009.

[11] S.-J. Bi, S.-H. Pan, and J.-S. Chen, "Nonsingularity conditions for the Fischer-Burmeister system of nonlinear SDPs," SIAM Journal on Optimization, vol. 21, no. 4, pp. 1392-1417, 2011.

[12] J. Faraut and A. Korányi, Analysis on Symmetric Cones, Oxford University Press, New York, NY, USA, 1994.

[13] M. Fukushima, Z.-Q. Luo, and P. Tseng, "Smoothing functions for second-order-cone complementarity problems," SIAM Journal on Optimization, vol. 12, no. 2, pp. 436-460, 2002.
[14] S. M. Robinson, "Strongly regular generalized equations," Mathematics of Operations Research, vol. 5, no. 1, pp. 43-62, 1980.

[15] F. H. Clarke, Optimization and Nonsmooth Analysis, John Wiley \& Sons, New York, NY, USA, 1983, reprinted by Society for Industrial and Applied Mathematics, Philadelphia, Pa, USA, 1990.

[16] L. Q. Qi, “Convergence analysis of some algorithms for solving nonsmooth equations," Mathematics of Operations Research, vol. 18, no. 1, pp. 227-244, 1993.

[17] A. Fischer, "Solution of monotone complementarity problems with locally Lipschitzian functions," Mathematical Programming, vol. 76, no. 3, pp. 513-532, 1997.

[18] J.-S. Pang, D. Sun, and J. Sun, "Semismooth homeomorphisms and strong stability of semidefinite and Lorentz complementarity problems," Mathematics of Operations Research, vol. 28, no. 1, pp. 39-63, 2003.

[19] F. Alizadeh and D. Goldfarb, "Second-order cone programming," Mathematical Programmingy, vol. 95, no. 1, pp. 3-51, 2003.

[20] S. M. Robinson, "First order conditions for general nonlinear optimization," SIAM Journal on Applied Mathematics, vol. 30, no. 4, pp. 597-607, 1976.

[21] J. F. Bonnans and A. Shapiro, Perturbation Analysis of Optimization Problems, Springer, New York, NY, USA, 2000.

[22] F. Facchinei, A. Fischer, and C. Kanzow, "Regularity properties of a semismooth reformulation of variational inequalities," SIAM Journal on Optimization, vol. 8, no. 3, pp. 850-869, 1998. 


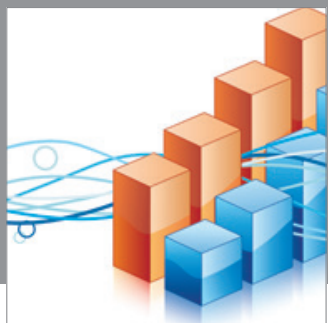

Advances in

Operations Research

mansans

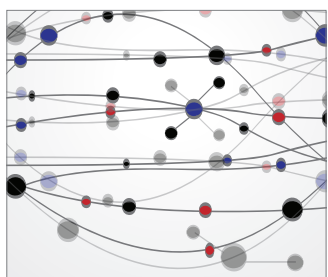

The Scientific World Journal
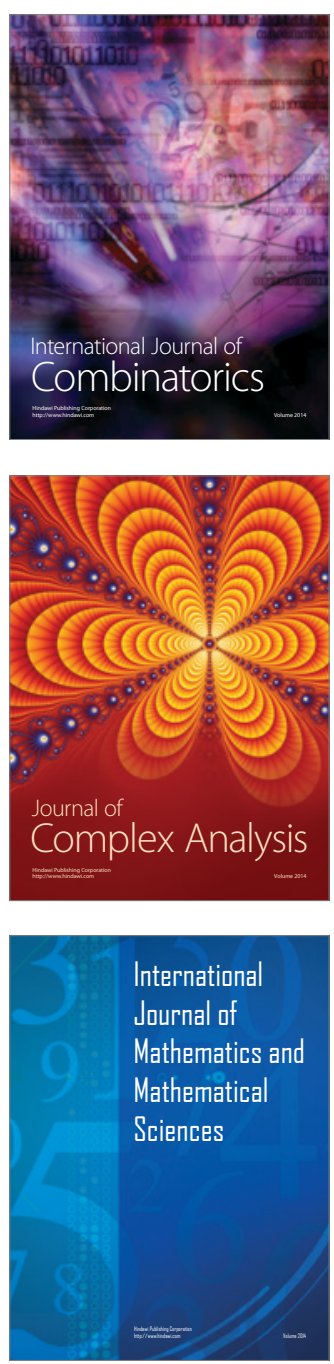
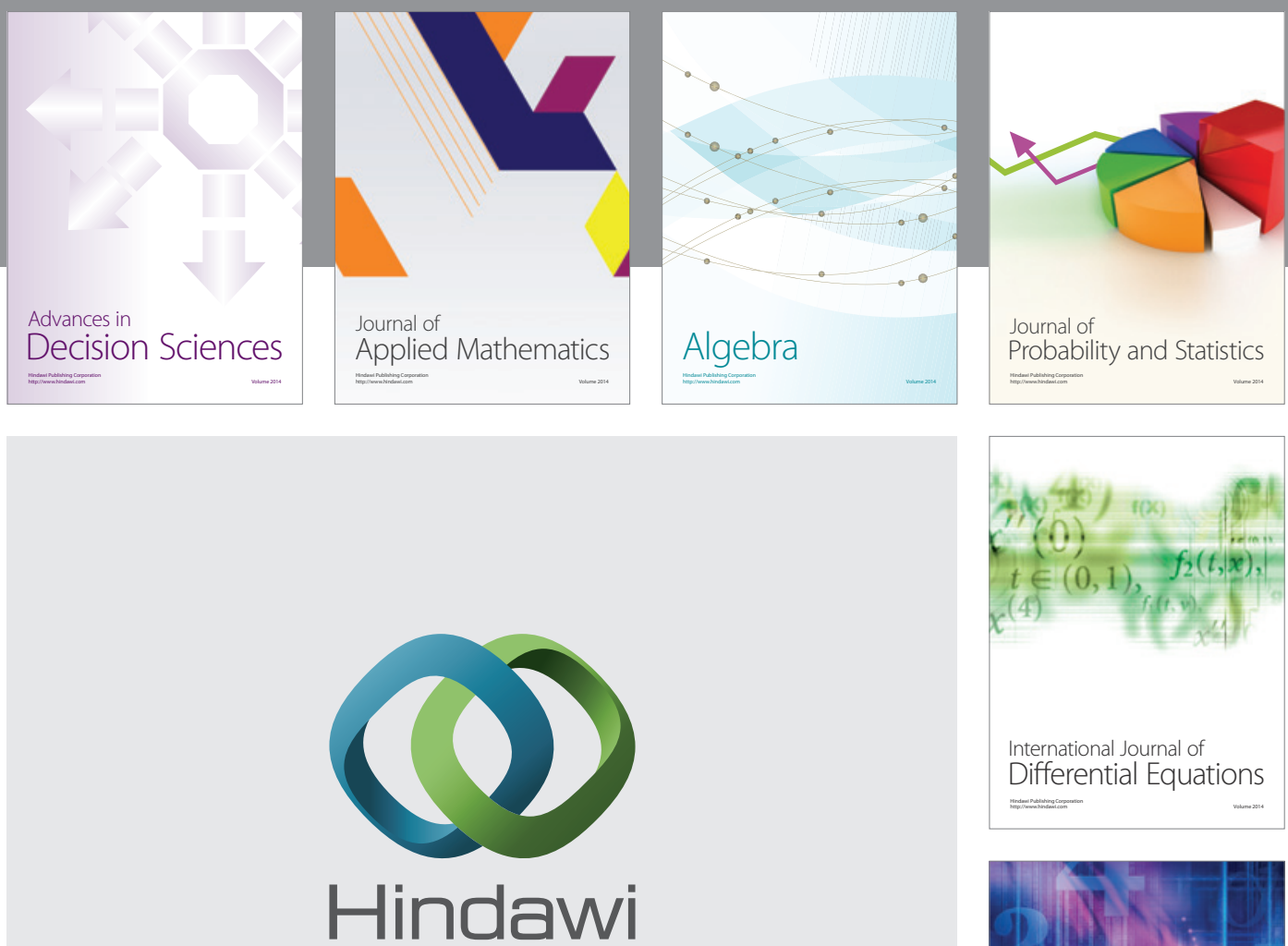

Submit your manuscripts at http://www.hindawi.com
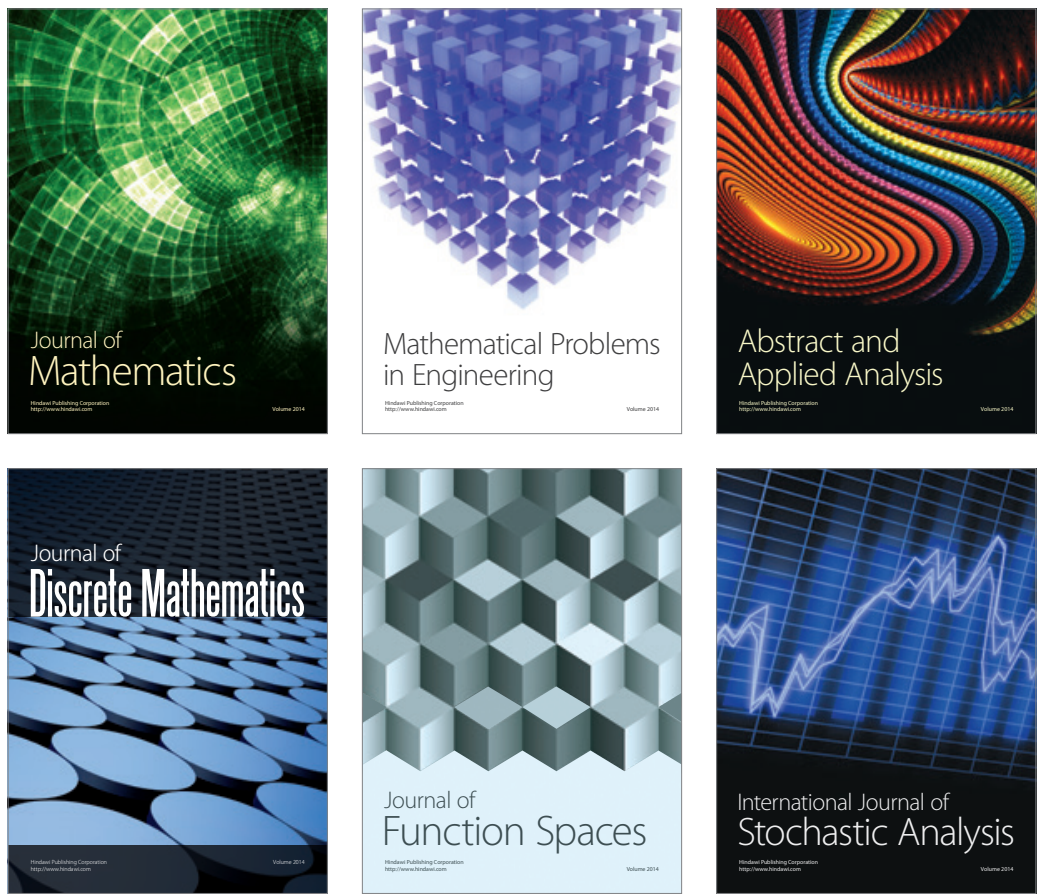

Journal of

Function Spaces

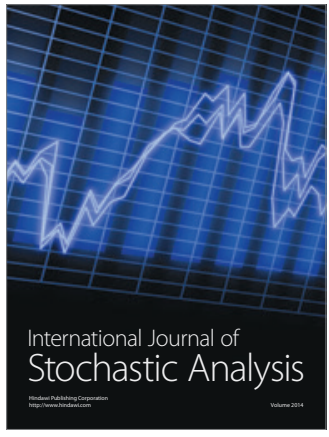

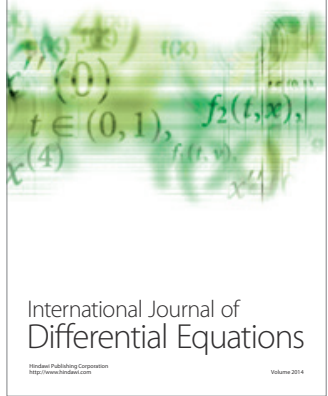
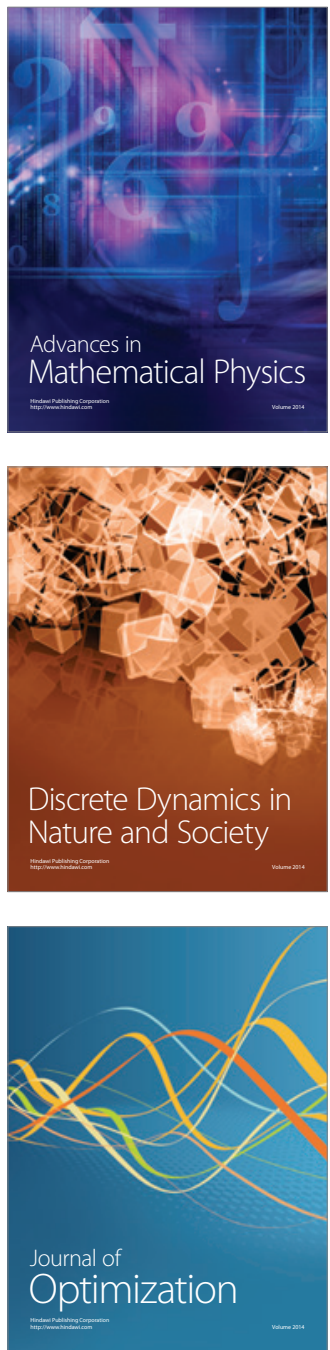